\title{
PUBLICACIONES SOBRE FILOLOGÍA INGLESA EN ESPAÑA (2008)
}

\section{RESUMEN}

Bibliografía relativa a Filología Inglesa publicada en España en 2008.

PALABRAS ClaVE: Filología Inglesa; bibliografía; España.

\section{AbSTRACT}

Bibliography on English Studies published in Spanish, 2008.

KEY WORDS: English Studies; bibliography; Spain.

ÍNDICE GENERAL
A. Literatura INGLESA
B. LITERATURA NORTEAMERICANA
C. OtRAS LITERATURAS EN LENGUA INGLESA
D. TRADUCCIÓN
E. Cultura y Cine 


\title{
A. LITERATURA INGLESA
}

\author{
ISABEL MEDRANO \\ mmedrano@flog.uned.es \\ ANA ZAMORANO \\ aizamorano@flog.uned.es \\ UNED. Madrid
}

ÍNDICE

1. Literatura anglosajona, medieval y renacentista

2. Restauración y siglo XVIII

3. Siglo XIX

4. Siglo XX y época contemporánea

5. Miscelánea

\section{LITERATURA ANGLOSAJONA, MEDIEVAL Y RENACENTISTA}

Carretero GonzÁlez, Margarita. «The Sound of Jealousy: Ot(h)ello between Shakespeare and Verdi». Studies in Honour of Neil Mclaren: A Man for all Seasons. Coord. Angeles Linde López, Juan Santana Lario y Celia Margaret Wallhead Salway. Granada: Editorial Universidad de Granada, 2008. 257-68.

Cerezo Moreno, Marta. «Roman Shakespeare and the Elizabethan View of the Roman Empire». Estudios de Filología Inglesa. Homenaje a la Dra. Asunción Alba Pelayo. Ed. Teresa Gibert Maceda y Laura Alba Juez. Madrid: UNED, 2008. 359-72.

Díaz Santiago, María Victoria. «The Necromancer Friar Bacon in the Magic World of Greene's Comedy Friar Bacon and Friar Bungay». SEDERI 18 (2008): 5-26.

Fernández Rodríguez, Carmen María. «Frail Patriarchy and the Authority of the Repressed in William Shakespeare's Measure for Measure». SEDERI 18 (2008): 27-44.

HERNÁNDEZ PÉREZ, María Beatriz. «Both Human and Divine: the Conflict between Confession and Gossip in The Book of Margery Kempe». SELIM 14 (2007). CD-ROM.

KRUMMrICH, Philip. «Jacks en las cajas: el potencial cómico de las escenas de baúl en Boccaccio, Shakespeare y Joanot Martorell». / «Jacks in the Boxes: the Comic Potential of the Chest Scenes in Boccaccio, Shakespeare, and Joanot Martorell». The Grove. Working Papers on English Studies 15 (2008): 29-40.

Marín CALVARRo, Jesús Ángel. «Ambigüedad y traducción: el soneto XII de Shakespeare / Ambiguity and Translation: Shakespeare's Sonnet XII». Revista de Filología de la Universidad de la Laguna 26 (2008): 103-16.

MARTíNEZ-DuEÑAs ESPEJO, José Luis. «El género epistolar y la retórica política del siglo XVI: The copy of a letter lately written by a Spanishe gentleman y su traducción al español». Studies in Honour of Neil Mclaren: A Man for all Seasons. Coord. Angeles Linde López, Juan Santana Lario y Celia Margaret Wallhead Salway. Granada: Editorial Universidad de Granada, 2008. $307-28$. 
Matuska, Agnes. «'Masking Players, Painted Sepulchers and Double Dealing Ambidexters' on Duty: anti Theatricalist Tracts on Audience Involvement and the Transformative Power of Plays». SEDERI 18 (2008): 45-60.

MONTERREY, Tomás. «'The Castalian Liquor’: Goce y mitificación del vino canario en la literatura inglesa». Estudios de Filología Inglesa. Homenaje a la Dra. Asunción Alba Pelayo. Ed. Teresa Gibert Maceda y Laura Alba Juez. Madrid: UNED, 2008. 459-69.

NúÑ̃z MËNDEZ, Eva. A Spanish Version of Troilus and Criseyde /Versión española de Troilus and Criseyde de Chaucer. The Edwin Mellen Press, 2008.

PÉrez Fernández, Tamara y SÁez Hidalgo, Ana. «'A Man Textueel’: Scribal Readings and Interpretations of Troilus and Criseyde through the Glosses in Manuscript British Library Harley 2392». SELIM 14 (2007). CD-ROM.

Ribes TraVer, Purificación. «Stefan Zweig's Volpone, eine lieblose Komödie: a Reassessment». SEDERI 18 (2008): 61-80.

RodRÍGUEZ RodríGUEZ, Beatriz María. «David Rowland's Lazarillo de Tormes (1586): Analysis of Expansions in an Elizabethan Translation». SEDERI 18 (2008): 81-96.

\section{RESTAURACIÓN Y SIGLO XVIII}

Altuna García de Salazar, Asier. «Robert Jephson: anglo-irlanda, un Lazarillo de Valencia y el recurso fársico a la comida en Two Strings to your Bow (1791)». / «Robert Jephson: AngloIreland, a Spanish Lazarillo of Valencia and the Farcical Recourse to Food in Two Strings to Your Bow (1791)». Odisea. Revista de Estudios Ingleses 9 (2008): 7-21.

DíAz BILD, Aída. «The Old Manor House and A Simple Story: A Harsh Lesson on Women's Defencelessness». Estudios de Filología Inglesa. Homenaje a la Dra. Asunción Alba Pelayo. Ed. Teresa Gibert Maceda y Laura Alba Juez. Madrid: UNED, 2008. 373-83.

EsTABLIER PÉREZ, Helena. «El matrimonio intelectual de Inés Joyes y Samuel Johnson: la traducción española de Rasselas, príncipe de Abisinia en la ideología de género de la Ilustración». Actas XIII Encuentro de la Ilustración al Romanticismo (1750-1850). Corod. María del Carmen García Tejera. Cádiz: Universidad de Cádiz, 2007. 443-454.

Galván, Fernando. «The Eighteenth-Century English Novel and its Spanish Heritage and Reception». Revista canaria de estudios ingleses 56 (April 2008): 123-44.

GUTIÉRREZ SuMILLERA, Rocío. «Tristram's Identity Revisited». Babel A.F.I.A.L., 17 (2208): 77-98.

MEDRANO ViCARIO, Isabel. «'Softened by tenderness'?: Sentimiento y ambigüedad en la obra de Frances Sheridan y Mary Wollstonecraft». Estudios de Filología Inglesa. Homenaje a la Dra. Asunción Alba Pelayo. Ed. Teresa Gibert Maceda y Laura Alba Juez. Madrid: UNED, 2008. 445-57.

Moya, Ana, LóPez, Gemma y Hurtley, Jacqueline A., ed., notas e intro. Pride and Prejudice: A Novel by Jane Austen . Barcelona: Publicacions i Edicions de la Universitat de Barcelona, 2008 (1st published, 2004).

Thomson, James. Winter. Edición, introducción, estudio y traducción versificada de Ángeles García Calderón. Almería: Editorial Universidad de Almería, 2007.

\section{SIGLO XIX}

AHRENS, Rüdiger. «Monstruosidad como ampliación imaginativa de la naturaleza humana: la poesía de Samuel Taylor Coleridge». / «Monstrosity as an imaginative extension of human nature: the poetry of Samuel Taylor Coleridge». The Grove. Working Papers on English Studies 15 (2008): 9-27. 
Ballesteros, Antonio. «La región más tenebrosa: Carlos Fuentes y su interpretación del mito de Drácula en Vlad». Reescrituras de los mitos en la literatura. Coord. Juan Herrero Cecilia y Montserrat Morales Peco. Cuenca: Servicio de Publicaciones de la Universidad de Castilla-La Mancha, 2008. 529-45.

- . «El laberinto femenino de la soledad y la fantasía: cuatro escritoras victorianas frente a lo sobrenatural». Sites of Female Terror: En torno a la mujer y el terror. Ed. Antón-Pacheco Bravo et al. Madrid: Editorial Aranzadi, 2008.41-64.

-. «El enmascaramiento gótico del deseo lésbico: los relatos sobrenaturales de Vernon Lee». Que sus faldas son ciclones: Representación literaria contemporánea del lesbianismo en lengua inglesa. Ed. Rosa García Rayego y M ${ }^{a}$ Soledad Sánchez Gómez. Madrid: Editorial Egales, 2008. 27-42.

EgEA FernÁNDEZ-Montesinos, Alberto. Viajeras románticas en Andalucía. Una antología. Centro de Estudios Andaluces, 2008.

Fernández Rodríguez, Carmen María. «From Hays to Burney: an Approach to Female Difficulties in Early Nineteenth-Century English Fiction». Estudios Ingleses de la Universidad Complutense 16 (2008): 51-66.

-. «Un oriente muy poco convencional: 'Murad the Unlucky' de Maria Edgeworth y su traducción al francés y al castellano en el siglo XIX». Sendebar: Boletín de la EUTI de Granada, 19 (2008):77-98.

Flores Moreno, Cristina. Plastic Intellectual Breeze. The Contribution of Ralph Cudworth to S. T. Coleridge's Early Poetics of the Symbol. Bern: Peter Lang, 2008.

Galván, Fernando. «Elegías y anti-elegías: El caso de Death de John Clare, o una expectativa frustrada». Estudios de Filología Inglesa. Homenaje a la Dra. Asunción Alba Pelayo. Ed. Teresa Gibert Maceda y Laura Alba Juez. Madrid: UNED, 2008. 393-408.

Guimarães, Paula Alexandra. «Dramatising the Conflicts of Nation and the Body: Displacement in Charlote and Emily Brontë's Poetry of 'Home' and 'Exile' Dualities». Miscelánea 38 (2008). Revista electronica.

Montes, Catalina. «La España decimonónica vista por un inglés». Estudios de Filología Inglesa. Homenaje a la Dra. Asunción Alba Pelayo. Ed. Teresa Gibert Maceda y Laura Alba Juez. Madrid: UNED, 2008.

RuIz Más, José. «Médicos-viajeros y viajeros-convalecientes de habla inglesa en la España mediterránea durante el siglo XIX». Epos XXIV (2008): 175-891.

\section{SigLO XX Y CONTEMPORÁNEA}

ACquarone, Cecilia. «Barriers, Borders and Crossings in two Postmodern Novels: Caryl Phillps's The Nature of Blood and Zadie Smith's White Teeth». Epos XXIV (2008): 207-21.

Aliaga RodRIGo, Esther. «Robert McLiam Wilson's Eureka Street: Real or Imagined Northern Ireland». The Irish Knot: Essays on Imaginary/Real Ireland. Ed. María José Carrera, Anunciación Carrera, Enrique Cámara, Celsa Dapía. Valladolid: Universidad de Valladolid, 2008. 65-70.

ANDRÉs CUEVAS, Isabel M${ }^{\mathrm{a}}$. «The King of Fools and the Bishop of Unreason: Virginia Woolf's Carnivalesque Vision in Between the Acts». Babel A.F.I.A.L. 17 (2008): 7-22.

-. «'We are [also] the hollow men': Unmasking Male Heroes in Jacob's Room». Studies in Honour of Neil Mclaren: A Man for all Seasons. Coord. Angeles Linde López, Juan Santana Lario y Celia Margaret Wallhead Salway. Granada: Editorial Universidad de Granada, 2008. 221-40.

- . «The Donkey-God and the Holy Stake: The Subversion of Religion Through Carnival Identities in Virginia Woolf's Between the Acts». Recycling Culture(s). Ed. Sara Martín Alegre. Cambridge Scholars Press, 2008. 3-11. 
-. «Monstruos, animales y otras féminas: la representación del cuerpo grotesco en las novelas de Virginia Woolf» Cuerpos de Mujeres: Miradas, Representaciones e Identidades. Ed Carmen Gregorio, Adelina Sánchez Espinosa y Ana M ${ }^{\mathrm{a}}$ Muñoz. Granada: Colección Feminae, Editorial Universidad de Granada, 2008. 189-204.

BALLESTEROS, Antonio. «El enmascaramiento gótico del deseo lésbico: los relatos sobrenaturales de Vernon Lee». Que sus faldas son ciclones: Representación literaria contemporánea del lesbianismo en lengua inglesa. Ed. Rosa García Rayego y María Soledad Sánchez Gómez. Madrid: Editorial Egales, 2008. 27-42.

Bayón García, Cristina y Fernández Bahillo, Héctor. «Between Biographical Footprints and Legendary Traces: The Real and Imaginary Worlds of At Swim-Two Birds by Flann O'Brien». The Irish Knot: Essays on Imaginary / Real Ireland. Ed. María José Carrera, Anunciación Carrera, Enrique Cámara, Celsa Dapía. Valladolid: Universidad de Valladolid, 2008. 363-72.

Calvo Maturana, María del Coral. «Edwin Morgan's Strawberries: Simbología y temporalidad». Studies in Honour of Neil Mclaren: A Man for all Seasons. Coord. Angeles Linde López, Juan Santana Lario y Celia Margaret Wallhead Salway. Granada: Editorial Universidad de Granada, 2008. 241-56.

CÁMARA ARENAS, Enrique y CALDERón QuINDÓs, Teresa. «Yeats's Theatrical Thinking: Imagination between the Real and the Realist». The Irish Knot: Essays on Imaginary / Real Ireland. Ed. María José Carrera, Anunciación Carrera, Enrique Cámara, Celsa Dapía. Valladolid: Universidad de Valladolid, 2008. 137- 47.

CARriedo, Lourdes, Guerrero, $\mathrm{M}^{\mathrm{a}}$ Luisa, MÉndez, Carmen y Vericat, Fabio, eds. A vueltas con Beckett. Madrid: La Discreta, 2008.

CONCHA MuÑoz, Ángeles de la. «La escritura de Jeanette Winterson: un desafío a la categorización y a las convenciones». Que sus faldas son ciclones: Representación literaria contemporánea del lesbianismo en lengua inglesa. Ed. Rosa García Rayego y $\mathrm{M}^{\mathrm{a}}$ Soledad Sánchez Gómez. Madrid: Editorial Egales, 2008. 131-48.

CONRAD, Joseph. El corazón de las tinieblas. Ed., trad. y notas D. López García. Madrid: Valdemar, 2008.

DaÑoBeITIA, María Luisa. «Eveline y la trasgresión femenina en la negación». Studies in Honour of Neil Mclaren: a Man for all Seasons. Coord. Angeles Linde López, Juan Santana Lario y Celia Margaret Wallhead Salway. Granada: Editorial Universidad de Granada, 2008. 269-78.

Estévez SAÁ, José Manuel. «A ‘True Believer’: Joseph O’Connor Imagining Dublin in the Second Half of the Twentieth Century». The Irish Knot: Essays on Imaginary/Real Ireland. Ed. María José Carrera, Anunciación Carrera, Enrique Cámara, Celsa Dapía. Valladolid: Universidad de Valladolid, 2008. 71-78.

FERNÁNDEZ SÁNCHEZ, José Francisco. «They took the dull coast road home’: Images of Ireland in Samuel Beckett's Dream of Fair to Middling Women. The Irish Knot: Essays on Imaginary / Real Ireland. Ed. María José Carrera, Anunciación Carrera, Enrique Cámara, Celsa Dapía. Valladolid: Universidad de Valladolid, 2008. 329-38.

FERNÁNDEZ-CAPARRÓS TURINA, Ana. «The influence of jazz and the reconstruction of the imagination in Sam Shepard's Suicide in B-Flat: creating 'a new dimension' in form and perception». Estudios Ingleses de la Universidad Complutense 16 (2008): 67-88.

GaLÁN MoYA, Rafael. «Anhelo, crítica y epifanía lésbica en Virginia Woolf». Que sus faldas son ciclones: Representación literaria contemporánea del lesbianismo en lengua inglesa. Ed. Rosa García Rayego y Ma Soledad Sánchez Gómez. Madrid: Editorial Egales, 2008. 57-78

García Landa, José Angel. «Atonement and Adaptation (on Ian McEwan's Novel and Joe Wright's Film)». (2008). Available at SSRN: http://ssrn.com/abstract=1091883.

- . «Hemingway Meets Beckett: Cormac McCarthy's The Road». (2008). Available at SSRN: http://ssrn.com/abstract=1085493. 
García León, Rafael I. «Joyce...Bruno...Ulysses». Odisea: Revista de estudios ingleses 9 (2008): 83-92.

GARCíA RAYEGO, Rosa. «'Eres una lámpara que arde para mí’: una aproximación a Radclyffe Hall». Que sus faldas son ciclones: Representación literaria contemporánea del lesbianismo en lengua inglesa. Ed. Rosa García Rayego y $\mathrm{M}^{\mathrm{a}}$ Soledad Sánchez Gómez. Madrid: Editorial Egales, 2008. 43 -56.

García TorTosa, Francisco. «The Backdrop of Translating Ulysses». Papers on Joyce 14 (2008): 27-38.

García Zarranz, Libe. «Classical Fairy Tales in Modern Landscapes: Felicia's Journey, by William Trevor». The Irish Knot: Essays on Imaginary / Real Ireland. Ed. María José Carrera, Anunciación Carrera, Enrique Cámara, Celsa Dapía. Valladolid: Universidad de Valladolid, 2008. 193-200.

GonZÁLEZ RodRíGUEZ, Luisa $\mathrm{M}^{\mathrm{a}}$. «La subversión del género policiaco en The Enigma, de John Fowles». Babel A.F.I.A.L. 17 (2208): 59-75.

HenRíQueZ JimÉNEZ, Santiago J. «Miguel de Unamuno y Graham Greene: Coincidencia en torno a los cuidados de la fe». Estudios de Filología Inglesa. Homenaje a la Dra. Asunción Alba Pelayo. Ed. Teresa Gibert Maceda y Laura Alba Juez. Madrid: UNED, 2008. Estudios de Filología Inglesa. Homenaje a la Dra. Asunción Alba Pelayo. Ed. Teresa Gibert Maceda y Laura Alba Juez. Madrid: UNED, 2008. 421-30.

Herrero Martín, Rosana. «Language Performativity in Donal O'Kelly's Catalpa (1995) and Enda Walsh's Disco Pigs (1996): Two Radical Theatre Proposals». The Irish Knot: Essays on Imaginary / Real Ireland. Ed. María José Carrera, Anunciación Carrera, Enrique Cámara, Celsa Dapía. Valladolid: Universidad de Valladolid, 2008. 231-42.

-. The Doing of Telling on the Irish Stage. A Study of Language Performativity in Modern and Contemporary Irish Theatre. Frankfurt am Main: Peter Lang, 2008.

Herrero Quirós, Carlos. «Landscape as Text in Seamus Heaney: The Irish 'Songlines'». The Irish Knot: Essays on Imaginary / Real Ireland. Ed. María José Carrera, Anunciación Carrera, Enrique Cámara, Celsa Dapía. Valladolid: Universidad de Valladolid, 2008. 187-92.

HewitT, Elena Carolina. «La primera escansión poética de la obra teatral Waiting for Godot». Estudios Irlandeses, 3 (2008): 113-20.

JARAZO Álvarez, Rubén. «Imagining Ireland in Twentieth-Century Spanish Writers: Diasporic Imagination in the Works of Álvaro Cunqueiro». The Irish Knot: Essays on Imaginary / Real Ireland. Ed. María José Carrera, Anunciación Carrera, Enrique Cámara, Celsa Dapía. Valladolid: Universidad de Valladolid, 2008. 383-91.

LÁZARO, Alberto. «The History of the First Catalan Ulysses, by J. F. Vidal Jové». Papers on Joyce 14 (2008): 59-70.

LÓPEZ ROPERO, María Lourdes. «Colonial Flâneurs: the London Life-writing of Janet Frame and Doris Lessing». Inside Out: Women Negotiating, Subverting, Appropriating Public and Private Space. Ed. Teresa Gómez Reus y Aránzazu Usandizaga. Amsterdam / New York: Rodopi, 2008. 189-203.

Lorenzo Modia, María Jesús y Fernández MÉNDEZ, Cristina. «Imaginations of Irish Womanhood vs. Real Women in Mary Beckett's A Belfast Woman». The Irish Knot: Essays on Imaginary $/$ Real Ireland. Ed. María José Carrera, Anunciación Carrera, Enrique Cámara, Celsa Dapía. Valladolid: Universidad de Valladolid, 2008. 223-30.

LosADa PÉREz, Ana. «The Spectre of Ideology in Joyce's «The Dead». The Irish Knot: Essays on Imaginary / Real Ireland. Ed. María José Carrera, Anunciación Carrera, Enrique Cámara, Celsa Dapía. Valladolid: Universidad de Valladolid, 2008. 299-306.

LosAdA, J. M. «Las filosofías de Molloy». A vueltas con Beckett. Ed. Lourdes Carriedo, M ${ }^{a}$ Luisa Guerrero, Carmen Méndez y Fabio Vericat. Madrid: La Discreta, 2008. 43-54. 
MALlafré, Joaquim. «Translating Ulysses». Papers on Joyce 14 (2008): 71-84.

Martín Alegre, Sara. «La inquietud de Ulises: la crisis postbélica de la masculinidad en The Soldier's Return y A Son of War de Melvyn Bragg». / «Odysseus's Unease: The Post-war Crisis of Masculinity in Melvyn Bragg's The Soldier's Return and A Son of War». Odisea. Revista de Estudios Ingleses 9 (2008): 133-144.

Martín SALVÁN, Paula. «The great worth of the people': Models of Community in Joseph Conrad's Nostromo». Estudios Ingleses de la Universidad Complutense 16 (2008): 89-109.

Mateo Martínez, José. «El teatro de Hugh Whitemore: Un naturalismo necesario». Estudios de Filología Inglesa. Homenaje a la Dra. Asunción Alba Pelayo. Ed. Teresa Gibert Maceda y Laura Alba Juez. Madrid: UNED, 2008. 431-44.

MenÉndez Salmon, Ricardo. Travesías del mal: Conrad, Céline, Bolaño. Oviedo: Universidad de Oviedo, 2007.

Miquel BALDELlou, Marta. «Demonización de las heroínas victorianas que pasan a la madurez en Lucrecia de Edgard Bulwer-lytton y los cuentos de mujeres de Edgar Allan Poe». / «Demonising the Victorian Heroine's Coming-of-Age in Edward Bulwer-lytton's Lucretia and Edgar Allan Poe's Women's Tales». Odisea. Revista de Estudios Ingleses 9 (2008): 179-189.

- . "Coming of age inside, outside the big house, and beyond: a poetics of ageing and decay through John Banville's Birchwood.» Revista de Filología de la Universidad de la Laguna 26 (2008): $135-50$.

MonNiCKENDAM, Andrew. «Goodbye to Isherwood: the Rise and Fall of a Literary Reputation». Atlantis 30.2 (2008): 125-37.

Nicolás Román, Susana. La figura femenina en el teatro de Edward Bond: mujeres perdidas. Almería: Editorial Universidad de Almería, 2007.

- . Historia de una heroína: pacifismo y locura en The Woman de Edward Bond / A heroine's story: pacifism and madness in Edward Bond's The Woman». Revista de Filología de la Universidad de la Laguna 26 (2008): 181-90.

Nieto GaRcía, Jesús María. «Discourse, Interpretation and Teaching Literature: a close reading of Lawrence's Fanny and Annie over Twenty-five Years Later». Studies in Honour of Neil Mclaren: A Man for all Seasons. Coord. Angeles Linde López, Juan Santana Lario y Celia Margaret Wallhead Salway. Granada: Editorial Universidad de Granada, 2008. 329-38.

Olivares AllepuZ, $\mathrm{M}^{\mathrm{a}}$ de la Bendición. «Imagining a New Mother-Daughter Relation: Jennifer Johnston's Construction of the Female Servant in The Old Jest». The Irish Knot: Essays on Imaginary / Real Ireland. Ed. María José Carrera, Anunciación Carrera, Enrique Cámara, Celsa Dapía. Valladolid: Universidad de Valladolid, 2008. 263-70.

PACHeCo Costa, Verónica. «Mujer, cuerpo y religión en el cuento de A. S. Byatt The Djinn in the Nightingle's Eye». De lo sagrado y lo profano. Mujeres tras/entre/sin fronteras. Ed. Mercedes Arriaga Flórez et al. Sevilla: Arcibel Editores, 2008. 293-300.

PASCUAL SOLER, Nieves. "'No puedo ocuparme del asesino si me preocupo por no salir del armario': misterios y lesbianas». Que sus faldas son ciclones: Representación literaria contemporánea del lesbianismo en lengua inglesa. Ed. Rosa García Rayego y M ${ }^{a}$ Soledad Sánchez Gómez. Madrid: Editorial Egales, 2008. 203-16.

PASTOR Comín, Juan José. «Compositional Thought and Musical Structure in Joyce's Works / Proceder compositivo y estructura musical en las obras de Joyce». Estudios Ingleses de la Universidad Complutense 16 (2008): 111-27.

PÉREZ Simón, Andrés. «Estrategias de desfamiliarización en Travesties de Stoppard» / «Devices of estrangement in Stopard's Travesties». The Grove: Working Papers on English Studies 15 (2008): 111-23.

PIVIDORI, Cristina. «Out of the Dark Room: Photography and Memory in Rachel Seiffert's Holocaust Tales». Atlantis 30.2 (2008): 79-94. 
Ráez PAdilla, Juan. «'Keeping Going': Alquimia, violencia y sacrificio en la poesía de Seamus Heaney». Miscelánea 38 (2008). Revista electronica.

Ramos GAY, Ignacio. «Joe Orton and the Subversion of the French Farcical Tradition». Babel A.F.I.A.L. 17 (2208): 115-25.

Rosende PÉREZ, Aida. «Revisiting 'Real' Ireland: New (Imagi)Nations in Éilís Ní Dhuibhne's The Dancers Dancing». The Irish Knot: Essays on Imaginary / Real Ireland. Ed. María José Carrera, Anunciación Carrera, Enrique Cámara, Celsa Dapía. Valladolid: Universidad de Valladolid, 2008. 291-98.

SÁnchez CAlle, María Pilar. «The Artist as a Mongrel Girl: Mina Loy’s Anglo-Mongrels and the Rose». ES: Revista de filología inglesa 29 (2008): 167-83.

SALIS, Loredana. «The Sepulchral Metaphor in The Burial at Thebes, a Translation of Sophocles' Antigone by Seamus Heaney». The Irish Knot: Essays on Imaginary / Real Ireland. Ed. María José Carrera, Anunciación Carrera, Enrique Cámara, Celsa Dapía. Valladolid: Universidad de Valladolid, 2008. 207-13.

Sierra Ayala, Lina. «Times of War and Peace: Sequels and Phobias in Rebecca West's The Return of the Soldier». Estudios de Filología Inglesa. Homenaje a la Dra. Asunción Alba Pelayo. Ed. Teresa Gibert Maceda y Laura Alba Juez. Madrid: UNED, 2008. 503-14.

-. «La visión realista de Irlanda de Maria Edgeworth». The Irish Knot: Essays on Imaginary / Real Ireland. Ed. María José Carrera, Anunciación Carrera, Enrique Cámara, Celsa Dapía. Valladolid: Universidad de Valladolid, 2008. 339-46.

TORRES ZÚÑIGA, Laura. «Feminist commentary in film adaptation: an analysis of Shelmerdine in Sally Potter's Orlando». Periphery and Centre III. Ed Rubén Jarazo y Lidia M. Montero. La Coruña: Nino, 2008. 37-46.

Usandizaga, Aránzazu. Expanding the Private and Public Spaces of War: Vera Brittain's Testament of Youth». Inside Out: Women Negotiating, Subverting, Appropriating Public and Private Space. Ed. Teresa Gómez Reus y Aránzazu Usandizaga. Amsterdam / New York: Rodopi, 2008. 249-71

VILlaR Argálz, Pilar. "A courage the other side of despair: The hopeful stoicism of Samuel Beckett's work». Studies in Honour of Neil Mclaren: A Man for all Seasons. Coord. Angeles Linde López, Juan Santana Lario y Celia Margaret Wallhead Salway. Granada: Editorial Universidad de Granada, 2008. 363-70.

-. «A Postcolonial Reading of Seamus Deane's Reading in the Dark». The Irish Knot: Essays on Imaginary / Real Ireland. Ed. María José Carrera, Anunciación Carrera, Enrique Cámara, Celsa Dapía. Valladolid: Universidad de Valladolid, 2008. 271-82.

- . «Encuentro con el poeta irlandés Theo Dorgan: reflexiones sobre la poesía contemporánea en Irlanda». Ficciones: Revista de Letras 11-13 (2008):2-5.

Wallhead Salway, Celia Margaret. «Tesla and the Tunguska Event in Thomas Pynchon's Against the Day». Studies in Honour of Neil Mclaren: A Man for all Seasons. Coord. Angeles Linde López, Juan Santana Lario y Celia Margaret Wallhead Salway. Granada: Editorial Universidad de Granada, 2008. 371-88.

WARD, Dunstan (ed.), Fiol, Joan Miquel y Seguí, Juana María (ass.eds.). The Art of Collaboration: Essays on Robert Graves and His Contemporaries. Colección Estudis Anglesos, 1. Palma de Mallorca: Universitat de les Illes Ballears, 2008.

\section{MiscelÁNEA}

ANTÓN-PACHECO BRAVO, Ana et al. Sites of Female Terror: En torno a la mujer y el terror. Madrid: Editorial Aranzadi, 2008. 
Aritzi, Bárbara y Martínez-FAlquina, Silvia, eds. e intro. On the Turn: The Ethics of Fiction in Contemporary Narrative in English. Newcastle: Cambridge Scholars Publishing, 2007.

BALLESTEROS, Antonio. «El laberinto femenino de la soledad y la fantasía: cuatro escritoras victorianas frente a lo sobrenatural». Sites of Female Terror: En torno a la mujer y el terror. Ed. Antón-Pacheco Bravo et al. Madrid: Editorial Aranzadi, 2008. 41-64.

—. Novela Inglesa Contemporánea. Guía Didáctica. Madrid: UNED, 2008.

B ARbeito, J. Manuel, FeiJóo, Jaime, FigueroA, Antón y SACIDO, Jorge, eds. National Identities and European Literatures. Bern: Peter Lang, 2008.

- . «Literature and Identitary Processes: An Introduction». National Identities and European Literatures. Bern: Peter Lang, 2008. 7-30.

Barbeito, J. Manuel y SACIDO, Jorge . «The Ghost of the Empire and the 'English' Postcolonial Identity». National Identities and European Literatures. Bern: Peter Lang, 2008. 123-46.

CANEda CABrera, M. Teresa. «Polyglot Voices, Hybrid Selves and Foreign Identities: Translation as a Paradigm of Thought for Modernism» Atlantis. Journal of the Spanish Association of Anglo-American Studies 30.1 (2008): 53-67.

CARABí, Àngels y Josep M. Armengol, eds.: La masculinidad a debate. Barcelona: Icaria, 2008.

Diamante COLADO, Guillermo. «Las puertas abiertas. Otros universos: la ciencia-ficción lesbiana.» Que sus faldas son ciclones: Representación literaria contemporánea del lesbianismo en lengua inglesa. Ed. Rosa García Rayego y $\mathrm{M}^{\mathrm{a}}$ Soledad Sánchez Gómez. Madrid: Editorial Egales, 2008. 217-34.

EsPínOLA Rosillo, Carmen. «Her story: narrating the lives of female terrorists». Studies in Honour of Neil Mclaren: A Man for all Seasons. Coord. Angeles Linde López, Juan Santana Lario y Celia Margaret Wallhead Salway. Granada: Universidad de Granada, 2008. 289-96.

García Landa, José Angel. «Emergent Narrativity». (2008). Available at SSRN: http://ssrn.com/abstract=1099358.

- «B $\log s$ and the Narrativity of Experience». (April 2008). Available at SSRN: http://ssrn.com/abstract=1113321.

-. «Tomorrow Will Have Been Written». (October 7, 2008). Available at SSRN: http://ssrn.com/abstract=1279918

García RaYego, Rosa y SÁnchez Gómez, María Soledad, eds. Que sus faldas son ciclones. Representación literaria contemporánea del lesbianismo en lengua inglesa. Madrid: Editorial Egales, 2008.

Gibert Maceda, Teresa y Alba Juez, Laura, eds. Estudios de Filología Inglesa. Homenaje a la Dra. Asunción Alba Pelayo. Madrid: UNED, 2008.

Gómez Reus, Teresa y Usandizaga, Aránzazu eds., Inside Out: Women Negotiating, Subverting, Appropriating Public and Private Space. Ed. Teresa Gómez Reus y Aránzazu Usandizaga. Amsterdam / New York: Rodopi, 2008.

- . «Introduction». Inside Out: Women Negotiating, Subverting, Appropriating Public and Private Space. Ed. Teresa Gómez Reus y Aránzazu Usandizaga. Amsterdam / New York: Rodopi, 2008. 19-33.

Gutiérrez Arranz, José María, ed. A Multicultural and Multifaceted Study of Ideologies and Conflicts Related to the Complex Realities and Fictions of Nation and Identity Represented in Contemporary Literature Written in English. Newcastle: Cambridge Scholars Publishing, 2008.

Herrero Ruiz, Javier. «En el cruce entre literatura, cultura, lingüística y cognición: castigo y metáforas morales en los cuentos de hadas». / «At the Crossroads between Literature, Culture, Linguistics, and Cognition: Punishment and Moral Metaphors in Fairy Tales». Odisea. Revista de Estudios Ingleses 9 (2008): 117-13.

Linde LóPez, Ángeles, Santana LaRio, Juan y Wallhead Salway, Celia M., coord. Studies in Honour of Neil Mclaren: A Man for all Seasons. Granada: Universidad de Granada, 2008. 
Lorenzo Modia, María Jesús y Boland Osegueda, Roy C., eds. Australia and Galicia: Defeating the Tyranny of Distance / Australia e Galicia: vencendo a tiranía do afastamento. Sydney: Antipodas Monographs; Xunta de Galicia, 2008.

MEDRANO, Isabel et al. «Publicaciones sobre Filología Inglesa en España (2007). Literatura Inglesa». EPOS XXIV (2008): 307- 62.

MonNiCKENDAM, Andrew. «La mente y el cuerpo en la literatura de guerra y de la paz posterior»./ «The mind and the body in the literature of war and back to peace». The Grove. Working Papers on English Studies 15 (2008): 55-73.

NiETo García, Jesús María. «Discourse, interpretation and teaching literature: a close reading of Lawrence's Fanny and Annie over twenty-five years later». Studies in honour of Neil Mclaren: A Man for all Seasons. Coord. Angeles Linde López, Juan Santana Lario y Celia Margaret Wallhead Salway. Granada: Editorial Universidad de Granada, 2008. 329-338.

PIER, John, and García LANDA, José Angel, eds. Theorizing Narrativity. Berlín and New York: Walter de Gruyter, 2008.

Prado-Pérez, Jose Ramón y Llorens-Cubedo, Dídac, eds. New Literatures of Old. Dialogues of Tradition and Innovation in Anglophone Literature. Newcastle upon Tyne: Cambridge Scholars Publishing, 2008.

RODRíGUEZ GUERRERO-STRACHAN, Santiago. En torno a los márgenes. Ensayos de literatura poscolonial. Minotauro Digital, 2008.

SuÁrez Briones, Beatriz «Introducción: 'A Chloe le gustaba Olivia.' Acerca de la identidad y la escritura lesbianas». Que sus faldas son ciclones: Representación literaria contemporánea del lesbianismo en lengua inglesa. Ed. Rosa García Rayego y M ${ }^{\mathrm{a}}$ Soledad Sánchez Gómez. Madrid: Editorial Egales, 2008. 11-26.

VÁzqueZ y del Árbol, Esther y VÁzquez Marruecos, José Luis, eds. Poesía Escocesa. Antología Bilingüe. Granada: Universidad de Granada, 2007.

Walton, David: Introducing Cultural Studies: Learning through Practice. London: Sage Publications Ltd, 2008.

ZAMORANO, Ana. «Chicas con tablas: representación del cuerpo lesbiano en el teatro anglo-norteamericano». Que sus faldas son ciclones. Representación literaria actual del lesbianismo en lengua inglesa. Ed. Rosa García Rayego y Mª Soledad Sánchez Gómez, Madrid: Editorial Egales. 2008. 205-233. 


\section{B. LITERATURA NORTEAMERICANA}

ISABEL MEDRANO

mmedrano@flog.uned.es

ANA ZAMORANO

aizamorano@flog.uned.es

UNED. Madrid

Aguilera Linde, Mauricio D. «'Truth is Held in Disrepute': O. Henry and the Dismantling of Paradigms». Miscelánea 38 (2008). Revista electrónica.

Álvarez, María Antonia. «¿Autobiografía o puro cuento? (Re)construcción de la identidad chicana en Caramelo, de Sandra Cisneros». Estudios de Filología Inglesa. Homenaje a la Dra. Asunción Alba Pelayo. Ed. Teresa Gibert Maceda y Laura Alba Juez. Madrid: UNED, 2008. 333-45.

Álvarez Amorós, José Antonio. «A Reappraisal of Henry James's Ideas on Theatre and Drama». Estudios de Filología Inglesa. Homenaje a la Dra. Asunción Alba Pelayo. Ed. Teresa Gibert Maceda y Laura Alba Juez. Madrid: UNED, 2008. 313-31.

-. «La obra dramática de Henry James: una revisión crítica». / «Henry James’s Dramatic Drama: A Critical Account». Odisea. Revista de Estudios Ingleses 9 (2008): 23-37.

BALlesteros, Antonio. «Feeling with and in the Other: Empathy, Pain and the Holocaust in Art Spiegelman's Maus». Feeling in Others: Essays on Suffering and Empathy in Modern American Culture. Ed. Nieves Pascual y Antonio Ballesteros. Zurich \& Berlin: Lit Verlag, 2008. 14558.

BarRanCo UreÑa, Empar. Willa Cather. El reverso de la alfombra. Biblioteca Javier Coy d'Estudis Nord-Americans. Valencia: Universidad de Valencia, 2008.

BARrIo MARCO, José Manuel. «Indómito y genial Mailer». Nexus II (2008): 91-96.

BARrIos, Olga. The Black Theatre Movement in the United States and in South America. Biblioteca Javier Coy d'Estudis Nord-Americans. Valencia: Universidad de Valencia, 2008.

BRITO, Manuel. «Aventuras y respuestas: Las pequeñas revistas americanas de poesía: 19702000». Estudios de Filología Inglesa. Homenaje a la Dra. Asunción Alba Pelayo. Ed. Teresa Gibert Maceda y Laura Alba Juez. Madrid: UNED, 2008. 347-58.

Beltrán Llavador, Fernando y Pearson, Paul P., coords. Semillas de esperanza: el mensaje contemplativo de Thomas Merton. Cóbreces (Cantabria): CISTERCIUM; Ávila: Centro Internacional de Estudios Místicos (CIEM), 2008.

Bosch, Marta, Cuenca, Mercè, Miravet, Mònica y Seguro, Mª Isabel. «Representing Trauma in American Women's Literature». (Mesa redonda). Proceedings 31st AEDEAN Conference. Ed. María Jesús Lorenzo Modia. A Coruña: Universidade da Coruña, 2008. CD-ROM.

BurILLO, R. «Entropy and the Fantastic in Pynchon's Narratives». Comparative Literature and Culture. New Studies on the Fantastic in Literature 10. 4 (December 2008). Revista electrónica.

CaÑadas RodrígueZ, Emilo. "Orality and Other Southern Aspects in ZZ Packer's Short Stories». ES: Revista de filología inglesa 28 (2007-2008): 73-88.

-. «Norman Mailer and Truman Capote: A Brief Account of Parallel Lives». Nexus II (2008): 97104.

- . «Truman Capote's Early Short Stories or The Fight of a Writer to Find His Own Voice». Proceedings 31st AEDEAN Conference. Ed. María Jesús Lorenzo Modia. A Coruña: Universidade da Coruña, 2008. CD-ROM. 
Costa STAKSRUd, Liliana. «Hitos de la narrativa lesbiana desde los años sesenta: Sarah, Sarah y Rita Mae». Que sus faldas son ciclones: Representación literaria contemporánea del lesbianismo en lengua inglesa. Ed. Rosa García Rayego y $\mathrm{M}^{\mathrm{a}}$ Soledad Sánchez Gómez. Madrid: Editorial Egales, 2008. 99-112.

Cuenca, Mercé y Seguro, María Isabel. «'Making Something Out of Nothing': Lesbianism as Liberating Fantasy in The Children's Hour». Atlantis 30.1 (2008): 115-127.

FERNÁNDEZ RodRíGUEZ, Carolina. «Between Guerras and Carnales: Chicana Feminist Writers and their Search for a Room of their Own». Babel A.F.I.A.L. 17: 23-47.

FERNÁNDEZ SAMPEDRO, $\mathrm{M}^{\mathrm{a}}$ Gema. El viaje en la ficción norteamericana. Símbolos e identidades. Biblioteca Javier Coy d'Estudis Nord-Americans. Valencia: Universidad de Valencia, 2008.

Ferrús Antón, Beatriz. La monja de Ágreda. Historia y leyenda de la dama azul en Norteamérica. Biblioteca Javier Coy d'Estudis Nord-Americans. Valencia: Universidad de Valencia, 2008.

GiBERT, Teresa. «Subverting the Master Narrative of Heroic Conquest: Thomas King's A Coyote Columbus Story (1992)». Estudios de Filología Inglesa. Homenaje a la Dra. Asunción Alba Pelayo. Ed. Teresa Gibert Maceda y Laura Alba Juez. Madrid: UNED, 2008. 409-20.

Gómez Reus, Teresa y Lauber, Peter. «In a Literary No Man's Land: A Spatial Reading of Edith Wharton's Fighting France». Inside Out: Women Negotiating, Subverting, Appropriating Public and Private Space. Ed. Teresa Gómez Reus y Aránzazu Usandizaga. Amsterdam/New Cork: Rodopi, 2008. 205-28.

GonzÁlez Groba, Constante. On Their Own Premises: Southern Women Writers and the Homeplace. Biblioteca Javier Coy d'Estudis Nord-Americans. Valencia: Universidad de Valencia, 2008.

GuRPEGUI, José Antonio, ed., Interpreting the New Millennium. Newcastle: Cambridge Scholars Publishing, 2008.

HARPER, Frances E. W. Iola Leroy, o las sombras disipadas. Trad. Ángeles Carreres. Biblioteca Javier Coy d'Estudis Nord-Americans.Valencia: Universidad de Valencia, 2008.

Ibarrola-Armendariz, Aitor. «Short-Story Form and Diversity Management in ZZ Packer's Drinking Coffee Elsewhere». Proceedings 31st AEDEAN Conference. Ed. María Jesús Lorenzo Modia. A Coruña: Universidade da Coruña, 2008. CD-ROM.

JimÉNEZ Placer, Susana Ma. «Ghosts, Words, Memories and Stories in Katherine Anne Porter's 'Old Mortality' and Sandra Cisneros's The House on Mango Street». Proceedings 31st AEDEAN Conference. Ed. María Jesús Lorenzo Modia. A Coruña: Universidade da Coruña, 2008. CD-ROM.

Kerouac, Jack. Mexico City Blues. Sesenta Poemas. Trad. Rolando Costa Picazo. Biblioteca Javier Coy d'Estudis Nord-Americans. Valencia: Universidad de Valencia, 2008.

LóPEz Liquete, María Felisa. «Exotic Bodies in Melville's Typee». Proceedings 31st AEDEAN Conference. Ed. María Jesús Lorenzo Modia. A Coruña: Universidade da Coruña, 2008. CDROM.

LÓPEZ ROPERO, María Lourdes. «Trust them to Figure it Out”: Toni Morrison's Books for Children». ATLANTIS. Journal of the Spanish Association of Anglo-American Studies. 30.2 (2008): 43-57.

Madinabeitia, Mónika. «Stories from the New West: Frank Bergon's New Western Fiction». ES: Revista de filología inglesa 29 (2008): 117-31.

Martín Alegre, Sara. «Edgar y Chicho: La pasión por Poe en Historias para no dormir». Proceedings 31st AEDEAN Conference. Ed. María Jesús Lorenzo Modia. A Coruña: Universidade da Coruña, 2008. CD-ROM.

MENESES NARANJo, Juan. «Caminando en la frontera: hibridismo y modulación del yo en tres novelas chicanas canónicas». / «Walking down the Borderline: Hybridity and the Modulation of the Self in Three Canonical Chicano Novels». Odisea. Revista de Estudios Ingleses 9 (2008): 167-178. 
Martín SAlván, Paula. "'Where Everything Converges to a Point': Conspiracy as Narrative Model in Don DeLillo's Fiction». ES: Revista de filología inglesa 29 (2008): 133- 52.

MuÑoz LunA, Rosa. «The Bell Jar: Towards a Fading in Mind and Color». Babel A.F.I.A.L. 17 (2008): 99-113.

NADAL, Marita. «Aberrations, Instabilities and Mythoclasm in the Tales of Flannery O'Connor». Revista canaria de studios ingleses 57 (November 2008): 181-94.

OLIVER-Rotger, $\mathrm{M}^{\mathrm{a}}$ Antonia. «Teoría encarnada y pensamiento fronterizo en las autobiografías de Gloria Anzaldúa y Cherrie Moraga.» Que sus faldas son ciclones: Representación literaria contemporánea del lesbianismo en lengua inglesa. Ed. Rosa García Rayego y Mª Soledad Sánchez Gómez. Madrid: Editorial Egales, 2008. 167-84.

ORne JeWETt, Sarah. La tierra de los abetos puntiagudos. Trad. Paul S. Derrick y Juan López Gavilán. Biblioteca Javier Coy d'Estudis Nord-Americans. Valencia: Universidad de Valencia, 2008.

PAscual Soler, Nieves. "No puedo ocuparme del asesino si me preocupo por no salir del armario': misterios y lesbianas». Que sus faldas son ciclones: Representación literaria contemporánea del lesbianismo en lengua inglesa. Ed. Rosa García Rayego y M ${ }^{a}$ Soledad Sánchez Gómez. Madrid: Editorial Egales, 2008. 203-16.

Pascual, Nieves y Ballesteros, Antonio, eds. Feeling in Others: Essays on Suffering and Empathy in Modern American Culture. Zurich \& Berlin: LitVerlag, 2008.

Perkins GILman, Charlotte. Mujeres y economía. Un estudio sobre la relación económica entre hombres y mujeres como factor de la evolución social. Trad: Empar Barranco Ureña. Biblioteca Javier Coy d'Estudis Nord-Americans. Valencia: Universidad de Valencia, 2008.

Petisco Martínez, Sonia, «'With the World in My Bloodstream': Thomas Merton's Wisdom of Love». Proceedings 31st AEDEAN Conference. Ed. María Jesús Lorenzo Modia. A Coruña: Universidade da Coruña, 2008. CD-ROM.

PiÑa Rosales, Gerardo. «El 'Quijote' en la literatura norteamericana». The Grove: Working Papers on English Studies 15 (2008): 125-39.

PIÑERO GIL, Eulalia. «La décima musa: poética amorosa y modernismo en la obra de Amy Lowell, Djuna Barnes y Gertrude Stein». Que sus faldas son ciclones: Representación literaria contemporánea del lesbianismo en lengua inglesa. Ed. Rosa García Rayego y $\mathrm{M}^{\mathrm{a}}$ Soledad Sánchez Gómez. Madrid: Editorial Egales, 2008. 79-98.

Rodríguez Guerrero-Strachan, Santiago, GonZÁlez Moreno, Beatriz e IbáÑEz IbáÑEZ, José Ramón. «El cronotopo en el cuento romántico». (Mesa redonda). Proceedings 31st AEDEAN Conference. Ed. María Jesús Lorenzo Modia. A Coruña: Universidade da Coruña, 2008. CDROM.

Rowlandson, Mary. La verdadera historia del cautiverio y restitución de la señora Mary Rowlandson. Trad. Elena Ortells Montón. Biblioteca Javier Coy d'Estudis Nord-Americans. Valencia: Universidad de Valencia, 2008.

SÁNCHEZ GómEZ, Ma Soledad. «'Una poesía completamente nueva empieza aquî́: poesía lesbiana contemporánea en Estados Unidos». Que sus faldas son ciclones: Representación literaria contemporánea del lesbianismo en lengua inglesa. Ed. Rosa García Rayego y $\mathrm{M}^{\mathrm{a}}$ Soledad Sánchez Gómez. Madrid: Editorial Egales, 2008. 113-30.

SÁNCHEZ-PARDo GonZÁLEZ, Esther. «The Lure of the Object in Henry James's Fictions of Thwarted Desire: Reflections on the Libidinal and Social Poetics of Literary Forms» Atlantis. Journal of the Spanish Association of Anglo-American Studies. 30.2 (2008): 27-41.

SANCHO GuINDA, Carmen. «An ethnological approach to Sister Nations' Verse: New Insights into Native American De-colonial Discourse». Revista Alicantina de Estudios Ingleses 21 (2008): 103-28.

SANTAYANA, George, La razón en el arte y otros escritos de estética. Ed. y trad. Ricardo Miguel Alfonso. Epílogo de Fernando Savater. Madrid: Verbum, 2008. 
Sanz Alonso, Irene. «Science Fiction Magnifying Mirrors: Octavia Butler's Dawn and the Ethics of Genetic Manipulation». Proceedings 31st AEDEAN Conference. Ed. María Jesús Lorenzo Modia. A Coruña: Universidade da Coruña, 2008. CD-ROM.

Soto García, Isabel. The Dialectics of Diaspora: Memory, Location and Gender. Biblioteca Javier Coy D'Estudis Nord-Americans. Ed. Mar Gallego, Isabel Soto. Valencia: Publicacions de la Universitat de València, 2009.

-. «'White People to Either Side': Native Son and the Poetics of Space». The Black Scholar 39. 12 (2008): 23-27.

-. «'The Spaces Left': Ambivalent Discourses in Harriet Jacobs and Frederick Douglass». Loopholes and Retreats. African American Writers and the Nineteenth Century. Ed. John Gruesser, Hanna Wallinger. Berlin: LIT Verlag, 2009. 31-42.

Stevenson MuÑz, Rocío. «Abigail Williams as a Femme Fatale in The Crucible». ES: Revista de filología inglesa 28 (2007-2008): 213-23.

TALly, Justine. «124 is Haunted. The Ghost Story as 'Ghost Text' in Toni Morrison's Beloved». Estudios de Filología Inglesa. Homenaje a la Dra. Asunción Alba Pelayo. Ed. Teresa Gibert Maceda y Laura Alba Juez. Madrid: UNED, 2008. 515-27.

TORRES ZÚÑIGA, Laura. «'Don't quote instinct to me!': An overview of the correlation of hunger and sexual desire in Tennessee Williams's short fiction». Studies in Honour of Neil Mclaren: A Man for all Seasons. Coord. Angeles Linde López, Juan Santana Lario y Celia Margaret Wallhead Salway. Granada: Editorial Universidad de Granada, 2008. 351-362.

ZAMORANO, Ana. «Chicas con tablas: representación del cuerpo lesbiano en el teatro anglo-norteamericano». Que sus faldas son ciclones. Represanteción literaria actual del lesbianismo en lengua inglesa. Ed Rosa García Rayego y M ${ }^{\mathrm{a}}$ Soledad Sánchez Gómez, Madrid: Editorial Egales. 2008 


\section{OTRAS LITERATURAS EN LENGUA INGLESA}

ISABEL MEDRANO

mmedrano@flog.uned.es

ANA ZAMORANO

aizamorano@flog.uned.es

UNED. Madrid

\section{ESTUDIOS SOBRE LITERATURA AFRICANA}

LóPEz RodRíGUez, Marta Sofía. «En otro sitio, no aquí: las lesbianas (in)visibles». Que sus faldas son ciclones: Representación literaria contemporánea del lesbianismo en lengua inglesa. Ed. Rosa García Rayego y M ${ }^{\text {a }}$ Soledad Sánchez Gómez. Madrid: Editorial Egales, 2008. $149-68$.

Pagola Montoya, Irene. «Gender-Based Discrimination as a Trigger for Cultural Hybridity in Tsitsi Dangarembga's Nervous Conditions». Proceedings 31st AEDEAN Conference. Ed. María Jesús Lorenzo Modia. A Coruña: Universidade da Coruña, 2008. CD-ROM

Zarandona FernándeZ, Juan Miguel. «From Periphery to Center?: The Collector of Treasures by Bessie Head (1977): The Translation and (Mis)reconstruction of an African Woman's Identity in Spanish, Italian and French». ES: Revista de filología inglesa 28 (2007-2008): 241-63.

\section{ESTUDIOS SOBRE LITERATURA ASIÁTICA}

Aguilera Linde, Mauricio D. «Indra's Absurd Ambitions: Themes and Patterns in Gopinath Mohanty's Short Fiction». Studies in Honour of Neil Mclaren: A Man for all Seasons. Coord. Angeles Linde López, Juan Santana Lario y Celia Margaret Wallhead Salway. Granada: Editorial Universidad de Granada, 2008. 221-30.

Hand, Felicity, NAVArRo TeJERo, Antonia, O'ConNor, Maurice y Oliva, Juan Ignacio. «Is there a Koiné in Narrating Diasporic Experiences of «Indianness?». Proceedings 31st AEDEAN Conference. Ed. María Jesús Lorenzo Modia. A Coruña: Universidade da Coruña, 2008. CDROM.

Martos Hueso, María Elena. «Mujer, genealogía, historia: reconstrucciones de la familia y la nación en The Shadow Lines de Amitav Ghosh y Difficult Daughters de Manju Kapur». / «Woman, Genealogy, History: Deconstructions of Family and Nation in Amitav Ghosh's The Shadow Lines and Manju Kapur's Difficult Daughters». Odisea. Revista de Estudios Ingleses 9 (2008): 155-166.

NAVARro Tejero, Antonia. «Gender without Borders: An Interview with C.S. Lakshmi (Ambai)». Revista canaria de estudios ingleses 57 (November. 2008): 159-64.

OLIVA, Juan Ignacio. «Vínculos y ausencias del padre en la literatura postcolonial angloindia». Estudios de Filología Inglesa. Homenaje a la Dra. Asunción Alba Pelayo. Ed. Teresa Gibert Maceda y Laura Alba Juez. Madrid: UNED, 2008. 491-502. 


\section{ESTUDIOS SOBRE LITERATURA AUSTRALIANA Y NEOCELANDESA}

Fresno Calleja, Paloma. «The Dramatisation of Pacific Diaspora in Albert Wendt's The Songmaker's Chair». Proceedings 31st AEDEAN Conference. Ed. María Jesús Lorenzo Modia. A Coruña: Universidade da Coruña, 2008. CD-ROM.

MARTín SALVÁn, Paula. «Topografía de la negritud en la ficción de J.M. Coetzee». / «Topographies of Blankness in J.M. Coetzee's Fiction». Odisea. Revista de Estudios Ingleses 9 (2008): 145-153.

\section{ESTUDIOS SOBRE LITERATURA CANADIENSE}

Alonso Breto, María Isabel. «Of Resistant Mothers and Women Turned Goddesses: Marlene Nourbese Philip's Salmon Courage». Proceedings 31st AEDEAN Conference. Ed. María Jesús Lorenzo Modia. A Coruña: Universidade da Coruña, 2008. CD-ROM.

BÉJAR, Alba de. «Identity and Belonging in Hiromi Goto's Chorus of Mushrooms». Proceedings 31st AEDEAN Conference. Ed. María Jesús Lorenzo Modia. A Coruña: Universidade da Coruña, 2008. CD-ROM.

CARmona Rodríguez, Pedro M. «Beyond the Edge of the Century: the Postcolonial Imagination in the 'New' Fictions of the Canadas». Miscelánea 38 (2008). Revista electronica.

Collellmir Morales, Dolors. «Legendary Survivors in Tomson Highway's Kiss of the Fur Queen». Proceedings 31st AEDEAN Conference. Ed. María Jesús Lorenzo Modia. A Coruña: Universidade da Coruña, 2008. CD-ROM.

Cuder Domínguez, Pilar. «Margaret Atwood's Metafictional Acts: Collaborative Storytelling in The Blind Assassin and Oryx and Crake». Revista canaria de estudios ingleses 56 (April 2008): 57-68.

Darias BeAutell, Eva. «Where Has 'Real' Nature Gone, Anyway? Ecocriticism, Canadian Writing and the Lures of the Virtual». Revista canaria de estudios ingleses 56 (April 2008): 81-98.

- . «The Pedagogy of the Sasquatch: Imagining the Aboriginal without Feathers in Eden Robinson's Monkey Beach». Proceedings 31st AEDEAN Conference. Ed. María Jesús Lorenzo Modia. A Coruña: Universidade da Coruña, 2008. CD-ROM.

DíAZ DuEÑAS, Mercedes. «Characterization through Nationality in the Works of Contemporary Canadian Writers». Studies in Honour of Neil Mclaren: A Man for all Seasons. Coord. Angeles Linde López, Juan Santana Lario y Celia Margaret Wallhead Salway. Granada: Editorial Universidad de Granada, 2008. 279-88.

Doвrotт, Gretchen. «From Physical to Personal Frontiers: Catharine Parr Traill's 'Pioneer Woman' Redefined in Margaret Laurence's The Diviners». Estudios de Filología Inglesa. Homenaje a la Dra. Asunción Alba Pelayo. Ed. Teresa Gibert Maceda y Laura Alba Juez. Madrid: UNED, 2008. 385-92.

GIBERT, Teresa. «Subverting the Master Narrative of Heroic Conquest: Thomas King's A Coyote Columbus Story (1992)». Estudios de Filología Inglesa. Homenaje a la Dra. Asunción Alba Pelayo. Ed. Teresa Gibert Maceda y Laura Alba Juez. Madrid: UNED, 2008. 409-20.

GONZÁLEZ GÁNDARA, Jorge. «(Post) Colonial Embroidery: un estudio sobre la situación de los personajes en Of Customs and Excise and Rachna Mara». Proceedings 31st AEDEAN Conference. Ed. María Jesús Lorenzo Modia. A Coruña: Universidade da Coruña, 2008. CD-ROM.

GonZÁlez Mínguez, $\mathrm{M}^{\mathrm{a}}$ Teresa. «British Tradition and the Quest for Canadian Identity in Alice Munro's Narrative». Babel A.F.I.A.L. 17 (2008): 49-58.

HERNÁEZ LERENA, María Jesús. «Two Voices from Newfoundland: History and Myth Addressed by Maura Hanrahan and Paul Butler». Revista canaria de estudios ingleses 56 (April 2008): 99122. 
Llarena Ascanio, María Jesús, «'The Nature of My Belongingness': Diaspora in M.G. Vassanji and the South Asian Novel in Canada». Revista canaria de estudios ingleses 56 (April 2008): $27-42$.

OLIVA, Juan Ignacio. «Construcciones problemáticas del exilio en la escritura de mujeres indo-canadienses». / «Problematic Constructions of Exile in Indo-Canadian Women Writing». The Grove: Working Papers on English Studies 15 (2008): 95-109.

\section{ESTUDIOS SOBRE LITERATURA CARIBEÑA}

Casado Gual, Núria. «'Finding Another Face inside my Face’: the Semiotics of Mime in Edgar Nkosi White's Racialized Dramaturgies». Miscelánea 38 (2008). Revista electrónica. 


\section{ESTUDIOS DE TRADUCCIÓN}

Eva SAMANIEGo FERNÁNDEZ

UNED. Madrid

esamaniego@flog.uned.es

AlCINA, Amparo. «Translation technologies. Scope, tools and resources». Target 20.1 (2008): 79102.

ARregui BARRAgán, Natalia. «La monodia estilística en traducción literaria». Intertexto y Polifonía: Homenaje a $M^{a}$ Aurora Aragón. Coord. Flor María Bango de la Campa, Antonio Niembro Prieto, Emma Alvarez Prendes. Oviedo: Universidad de Oviedo, 2008. 1005-1012.

BAKER, Mona y Saldanha, Gabriela. Routledge Enciclopedia of Translation Studies. London: Routledge, 2008.

BANDIA, Paul F. «Fictionalising translation and multilingualism». Target 20.1 (2008): 164-169.

- . Translation as Reparation: Writing and Translation in Postcolonial Africa. Manchester: St Jerome Publishing, 2008.

BAUMGARTEN, Nicole. «Yeah, that's it!: Verbal Reference to Visual Information in Film Texts and Film Translations». Meta 53. 1 (2008): 6-25.

Bodelón GonZÁLEZ, M. Encarnación. «La violencia contra las mujeres y el derecho no-androcéntrico: pérdidas en la traducción jurídica del feminismo». Género, violencia y derecho. Coord. Patricia Laurenzo Copello, María Luisa Maqueda Abreu, Ana María Rubio Castro. Editorial Tirant lo Blanch, 2008. 275-300.

BoydEn, Michael. «Is Translation Studies too much about translation? A reply to Jan Blommaert». Target 20. 1 (2008): 149-157.

BROWNLIE, Siobhan. «Resistance and non-resistance to boundary crossing in translation research». Target 20. 2 (2008): 333-347.

Colominas, Carme. «Towards chunk-based translation memories». Babel 54. 4 (2008): 343354.

DAM-Jensen, Helle y Zethsen, Karen Korning. «Translator awareness of semantic prosodies». Target 20.2 (2008): 203-221.

Davis, Paul. Translation and the Poet's Life. The Ethics of Translating in English. Oxford University Press, 2008.

Díaz CinTas, Jorge (ed.). The Didactics of Audiovisual Translation. John Benjamins, 2008.

Díaz Peralta, Marina, PiÑERo, Gracia y García Domínguez, María Jesús. «Ideología y selección lingüística en los textos históricos. Las traducciones españolas de History of the Conquest of Mexico de W. H. Prescott». Babel 54.3 (2008): 251-267.

DíAZ PÉREZ, Francisco Javier. «Wordplay in film titles. Translating English puns into Spanish». Babel 54. 1 (2008): 36-58.

DrosDov DíEz, Tatiana. «Linguiística del texto, semiótica de la cultura y semántica de la traducción (experiencias de una traducción literaria)». Eslavística Complutense 8 (2008): 35-60.

DrucKerman, Pamela. Lust in Translation. London: Penguin, 2008.

Eco, Humberto y Mc EwEN, Alastair. Experiences in Translation. Toronto: University of Toronto, 2008.

FERnÁNDEZ PARRILla, Gonzalo. «El siglo XXI en primera persona: de la traducción de autobiografías como antídoto». La traducción factor de cambio. Coord. María José Hernández Guerrero, Salvador Peña Martín. Bern: Peter Lang, 2008. 77-92. 
FERNÁNDEZ RAMOS, José Alfredo. Las ciencias humanas en lengua alemana y su traducción al español: la caracterización nominal: análisis contrastivo y traducción de textos filosóficos del alemán al español. León: Junta de Castilla y León, 2008.

Fung Chang, Nam. «A missing link in Itamar Even-Zohar's theoretical thinking». Target 20.1 (2008): 135-148.

García Álvarez, Ana M. «La traducción y su didáctica: estado actual de la cuestión». La formación de traductores e intérpretes: aproximación interdisciplinar. Coord. Isabel Pascua Febles. Las Palmas: Servicio de Publicaciones de la Universidad de Las Palmas de Gran Canaria, 2008. 41-51.

García DE Toro, Cristina. «Describing Catalan-Spanish translation». Babel 54.4 (2008): 369-379.

Gelpí ARroyo, Cristina. «Aproximación a la lexicografía digital para la traducción jurídica». La lexicografía bilingue y didactica: ayer y hoy. Coord. Mar Campos Souto, Eva María González González, José Ignacio Pérez Pascual. A Coruña: Universidade da Coruña, 2008. 83-92.

GonzÁlez Rey, Isabel. A Multilingual Focus on Contrastive Phraseology and Techniques for Translation. Dr. Kovac Verlag, 2008.

HERNÁNDEZ, Belén. Memoria y traducción: Conversación con Piero Menarini. Murcia: Universidad de Murcia, 2008.

JIANG, Qiuxia. «Aesthetic Progression in Literary Translation». Meta 53. 4 (2008): 860-871.

JIE, Xi y ZHONG, Yong. «Locating users of interpretation in the court. An impact analysis of literal and meaningful renditions in a mock court situation». Babel 54.4 (2008): 327-342.

JIMÉNEZ BELLVER, Jorge. «Afijación en la traducción de la terminología de la telefonía y las comunicaciones móviles: prefijación y sufijación». Actas del I Congreso Internacional de Filología Hispánica, Jóvenes Investigadores. Coord. José Antonio Calzón García. Oviedo: Universidad de Oviedo, 2008. 177-186.

Ko, Leong. «Teaching Interpreting by Distance Mode: An Empirical Study». Meta 53.4 (2008): 814-840.

Koshinen, K. Translating Institutions: An Ethnographic Study of EU Translation. St. Jerome Publishing, 2008.

LATHROP, Tom. «¿Se necesita otra nueva traducción del Quijote en inglés?». Tus obras los rincones de la tierra descubren: Actas del VI Congreso Internacional de la Asociación de Cervantistas. Coord. Alexia Dotras Bravo. Centro de Estudios Cervantinos, 2008. 385-390.

LóPEZ FoLGADO, Vicente. «Peregrinos, vagabundos y caminantes: entre la traducción y la paráfrasis». Estudios en homenaje a José Luis Guijarro Morales. Coord. María Luisa Mora Millán. Cádiz: Servicio de Publicaciones de la Universidad de Cádiz, 2008. 161-174.

LóPez ViLLALBA, María. «La traducción ilustrada como acción revolucionaria». La traducción factor de cambio. Coord. María José Hernández Guerrero, Salvador Peña Martín. Bern: Peter Lang, 2008, 27-58.

Marrero Pulido, Vicente y García Domínguez, María Jesús. «Oralidad y escrituridad en la traducción del coloquio literario». La formación de traductores e intérpretes: aproximación interdisciplinar. Coord. Isabel Pascua Febles. Las Palmas: Servicio de Publicaciones de la Universidad de Las Palmas de Gran Canaria, 2008. 119-136.

Martín de León, Celia. «Skopos and beyond. A critical study of functionalism». Target 20. 1 (2008): 1-28.

Martín García, Adolfo. «The Circumscribed Infinites Scheme (CIS). A deconstructive approach to translating poetry». Target 20.1 (2008), 115-134.

MARTín RodríGUEZ, Manuel. «Noticia sobre una temprana traducción al español de la Teoría de los sentimientos morales de A. Smith». Homenaje a Antonio Domínguez Ortiz. Coord. Juan Luis Castellano Castellano, Miguel Luis López-Guadalupe Muñoz. Granada: Universidad de Granada, 2008. 595-600. 
MORILLAS GARCíA, Esther. «La traducción como enamoramiento y dependencia: Italia y la literatura estadounidense». La traducción factor de cambio. Coord. María José Hernández Guerrero, Salvador Peña Martín. Bern: Peter Lang, 2008. 115-130.

MoRINI, Massimiliano. «Outlining a new linguistic theory of translation». Target 20.1 (2008): 2951.

MunDAY, Jeremy. Introducing Translation Studies: Theories and Applications. Routledge, $2008\left(2^{\text {nd }}\right.$ ed.).

OrtTinen, Riitta. «From Thumbelina to Winnie-the-Pooh: Pictures, Words, and Sounds in Translation». Meta 53.1 (2008). 76-89.

Ortega ArJonilla, Emilio. «Luis Alonso Schökel, pionero de los estudios de traducción en lengua española: semblanza de un maestro». La traducción factor de cambio. Coord. María José Hernández Guerrero, Salvador Peña Martín. Bern: Peter Lang, 2008. 235-286.

Palacios-GonZÁlez, Manuela. «The gender of translation Irish poetry in Galician». Babel 54. 3 (2008). 268-280.

PASCUA Febles, Isabel y MARCELO WIRNITZER, Gisela. «La traducción de la literatura juvenil en el aula a través de dos originales: aleman e inglés». La formación de traductores e intérpretes: aproximación interdisciplinar. Coord. Isabel Pascua Febles, 2008. Las Palmas: Servicio de Publicaciones de la Universidad de Las Palmas de Gran Canaria, 2008. 11-25.

Pegenaute, Luis de, Decesaris, Janet y Tricás, Mercè. La traducción del futuro: Mediación lingüística y cultural en el siglo XXI. Barcelona: PPU S.A., 2008.

PenABAd OTERo, Luis. «La traducción del discurso como conformación del Tercer Espacio». Estudios de metodología de la lengua inglesa (IV). Coord. Leonor Pérez Ruiz, Isabel Pizarro Sánchez, Elena González-Cascos. Valladolid: Servicio de Publicaciones de la Universidad de Valladolid, 2008. 257-266.

PEREIRA, Nilce M. «Book Illustration as (Intersemiotic) Translation: Pictures Translating Words». Meta 53. 1 (2008): 104-119.

Petisco Martínez, Sonia. « Oh dulce huida! ¡ Oh vuelo jubiloso!: traducción y comentario de una selección poética de Thomas Merton». Semillas de esperanza: el mensaje contemplativo de Thomas Merton. Coord. Fernando Beltrán Llavador y Paul M. Pearson. Avila : Centro Internacional de Estudios Místicos, 2008. 65-84.

PinILLA MartíneZ, Julia. «De la 'traducción' de los glosarios científico-técnicos en el siglo XVIII y su contribución a la creación del léxico especializado». Intertexto y Polifonía: Homenaje a $M^{\text {a }}$ Aurora Aragón. Coord. Flor María Bango de la Campa, Antonio Niembro Prieto, Emma Alvarez Prendes. Oviedo: Universidad de Oviedo, 2008. 1059-1066.

PIZARRo SÁNCHEZ, Isabel. «El blended learning en la enseñanza de la traducción especializada». Innovación docente: docencia y TICs. Coord. Cristina Guilarte Martín-Calero. Valladolid: Universidad de Valladolid, 2008. 197-208.

POMMER, Sieglinde E. «No creativity in legal translation?». Babel 54. 4 (2008): 355-368.

PrICE, Joshua M. «Translating social science, good versus bad utopianism». Target 20.2 (2008): 348-364.

Profeti, María Grazia. «La traducción en escena: teatro áureo español y destinatario italiano». Territorios en red: prácticas culturales y análisis del discurso. Coord. Susana Díaz, Andrea Goin. Madrid: Biblioteca Nueva, 2008. 57-74.

RABAB'AH, Ghaleb Ahmed. «Communication strategies in translation». Babel 54.2 (2008): 97-109.

RAMÓN, Noelia y LABRADOR, Belén. «Translations of '-ly' adverbs of degree in an English-Spanish Parallel Corpus». Target 20. 2 (2008): 275-292.

RENNERT, Sylvi. «Visual Input in Simultaneous Interpreting». Meta 53.1 (2008): 204-217.

REY-Jouvin, Bernardette. «Problemas interculturales en la traducción de la gastronomía». La formación de traductores e intérpretes: aproximación interdisciplinar. Coord. Isabel Pascua Fe- 
bles. Las Palmas: Universidad de Las Palmas de Gran Canaria, Servicio de Publicaciones, 2008. 55-64.

RichART MARSET, Mabel. «Algunas reflexiones en torno a la traducción audiovisual». Teoría y práctica docente: actas del II Congreso Internacional de Lengua, Literatura y Cultura de E/LE. Coord. Jorge Martí Contreras. Valencia, 2008. 295-310.

Richart Marset, Mabel y PeláEz Malagón, José Enrique. «La traducción de términos artísticos: problemas interpretativos». Teoría y práctica docente: Actas del II Congreso Internacional de Lengua, Literatura y Cultura de E/LE. Coord. Jorge Martí Contreras. Valencia, 2008. 311-324.

RIOJA BARROCAL, Marta. «La traducción inglés-español de textos narrativos censurados (19621969)». Estudios de metodología de la lengua inglesa (IV). Coord. Leonor Pérez Ruiz, Isabel Pizarro Sánchez, Elena González-Cascos. Valladolid: Universidad de Valladolid, 2008. 243-256.

RISKU, Hanna y PIRCHER, Richard. «Visual Aspects of Intercultural Technical Communication: A Cognitive Scientific and Semiotic Point of View». Meta 53. 1 (2008): 154-166.

RodríGueZ EsPINOSA, Marcos y ACUÑa PARTAL, Carmen. «Traducción, represión y malditismo: las lecturas de Peter Pan de María Luz Morales, Terenci Moix y Leopoldo María Panero». La traducción factor de cambio. Coord. María José Hernández Guerrero, Salvador Peña Martín. Bern: Peter Lang, 2008. 59-76.

RoMÁN MíNGUEZ, Verónica. «Exploración didáctica de un encargo real de traducción: análisis de dificultades en la traducción de una escritura de compraventa de acciones». Sendebar 19 (2008): 151-166.

SÁNCHEZ, María T. «La traducción de folletos turísticos: ¿Qué calidad demandan los turistas?». $B a$ bel 54. 3 (2008): 296-297.

SANTOYO, Julio César. Historia de la traducción: Viejos y nuevos apuntes. León: Junta de Castilla y León, 2008.

SEguRA, Joaquín. «Traducción y traductores». Enciclopedia del español en los Estados Unidos: Anuario del Instituto Cervantes. Coord. Humberto López Morales. Instituto Cervantes: Santillana, 2008. 961-972.

SEla-ShefFy, Rakefet. «The Translators' Personae: Marketing Translatorial Images as Pursuit of Capital». Meta 53.3 (2008): 609-622.

Scott-Tennent, Christopher y GonZÁlez Davies, María. «Effects of Specific Training on the Ability to Deal with Cultural References in Translation». Meta 53.4 (2008): 782-797.

Sмiтн, Verónica. «Visual Persuasion: Issues in the Translation of the Visual in Advertising». Meta 53. 1 (2008): 44-61.

Socorro Trujillo, Karina. «Una tipología textual en la didáctica de la traducción especializada: Una propuesta para la traducción comercial». La formación de traductores e intérpretes: aproximación interdisciplinar. Ed. Isabel Pascua Febles. Las Palmas: Universidad de Las Palmas de Gran Canaria, Servicio de Publicaciones, 2008. 27-39.

Soto García, Isabel. «Englishing the Spanish, or Fleeing the Mother Tongue». Estudios de Filología Inglesa. Homenaje a la Dra. Asunción Alba Pelayo. Ed. Teresa Gibert Maceda y Laura Alba Juez. Madrid: Universidad Nacional de Educación a Distancia, 2008. 561-573.

TAIVAlKoSKI-ShILOv, Kristina. «Subtitling 8 Miles in three languages. Translation problems and translator licence». Target 20. 2 (2008): 249-274.

TORRESI, Ira. «Advertising: A Case for Intersemiotic Translation». Meta 53.1 (2008): 62-75.

VALDEÓN, Roberto A. «Anomalous news translation. Selective appropriation of themes and texts in the internet». Babel 54. 4 (2008): 299-326.

-. «Sociology of Translation». Babel 54.3 (2008): 287-290.

VALERo-GarcÉs, Carmen y MARTIN, Anne. Crossing Borders in Community Interpreting: Definitions and Dilemmas. Benjamins Translation Library 76. Amsterdam and Philadelphia: John Benjamins, 2008. 
VAndePITte, Sonia. «Remapping Translation Studies: Towards a Translation Studies Ontology». Meta 53.3 (2008): 569-588.

VV. AA. Estudios críticos de traducción de literatura infantil y juvenil. Tomo II: Análisis de las traducciones de obras inglesas y alemanas a las cuatro lenguas oficiales en España. Oviedo: Septem Ediciones, 2008.

VV. AA. La traducción: Hacia un encuentro de lenguas y culturas. México: Comunicarte Editorial, 2008.

WADENSJÖ, Cecilia. «In and Off the Show: Co-constructing 'invisibility' in an Interpreter-Mediated Talk Show Interview». Meta 53.1 (2008): 184-203.

WALLIS, Julian M.S. «Interactive Translation vs. Pre-Translation in TMs: A Pilot Study». Meta 53. 3 (2008). 623-629.

Yuste RodRIGO, Elia (ed.). Topics in Language Resources for Translation and Localisation. Amsterdam: John Benjamins, 2008.

Zarandona, Juan Miguel. «Two Different Translations of Tennyson's The Idylls of the King into Spanish: The Road From a Text-Oriented Model to a Functional Model of Translation». Meta 53.3 (2008). 490-506.

ZARO, Juan Jesús. Traductores y traducciones de literatura y ensayo (1835-1919). Albolote: Editorial Comares, 2008.

ZhigANG, Ma y XUdONG, Wu. «Interpreting performance under different task-planning conditions». Babel 54.3 (2008). 201-233. 


\section{E. CULTURA Y CINE}

Ma LuZ ARroyo VÁzQUEZ larroyo@flog.uned.es

ANTONIA SAGREdo SANTOS asagredo@flog.uned.es

UNED. Madrid

\section{ÍNDICE}

1. Cultura

2. Cine

\section{Cultura}

Armillas Vicente, José María. «Ayuda secreta y deuda oculta. España y la independencia de los Estados Unidos». Norteamérica a finales del siglo XVIII: España y los Estados Unidos. Ed. Emma Sánchez Montañés, Sylvia L. Hilton, Almudena Hernández Ruigómez e Isabel GarcíaMontón. Coord. Eduardo Garrigues López-Chicheri. Madrid: Fundación Consejo EspañaEstados Unidos y Marcial Pons, 2008. 171- 196.

Arroyo VÁzQueZ, M ${ }^{\mathrm{a}}$ Luz. «Mirando más allá de las fronteras. La experiencia de las pioneras afroamericanas». De lo sagrado y lo profano. Mujeres tras/entre/sin fronteras. Ed. Mercedes Arriaga Flórez et al. Sevilla: Arcibel Editores, 2008. 55-65.

- . «Frances Perkins y Hillary Rodham Clinton: Su contribución a la mejora de la legislación social estadounidense». Estudios de Filología Inglesa. Homenaje a la Dra. Asunción Alba Pelayo. Ed. Teresa Gibert Maceda y Laura Alba Juez. Madrid: UNED, 2008. 577-591.

- . «El mito presidencial en los Estados Unidos: un estudio de caso». Historia y representación de la cultura global. Ed. Carla Rodríguez González y Rubén Valdés Miyares. Oviedo: KRK Ediciones, 2008. 217-225.

-. «Mundos paralelos: Aprendizaje lingüístico e intercultural de la lengua inglesa a través del cine». 25 Years of Applied Linguistics in Spain: Milestones and Challenges. Eds. Rafael Monroy y Aquilino Sánchez. Murcia: Universidad de Murcia, 2008. 17-23.

- . «El poder de la oratoria: el impacto del discurso de Barack H. Obama en la opinión pública». EPOS XXIV (2008): 223-239.

- y Sagredo Santos, Antonia. Los Estados Unidos en sus documentos. Madrid: UNED, 2008.

AsHe, Geoffrey. Merlín. Historia y leyenda de la Inglaterra del rey Arturo. Barcelona: Editorial Crítica, 2007.

Amores CARREDANo, Juan Bosco, ed. Religión, herejías y revueltas sociales en Europa y América. Bilbao: Servicio Editorial de la Universidad del País Vasco, 2008.

BARTLETT, Enric R. Del pacto internacional de derechos económicos, sociales y culturales a los objetivos del milenio. Barcelona: J. M. Bosch, 2007.

BARRIo MARCo, José Manuel. La huella de Cervantes y del Quijote en la cultura anglosajona. Universidad de Valladolid. Secretariado de publicaciones, 2007.

Borge, Francisco J. A New World for a New Nation: the Promotion of America in Early Modern England. Bern: Peter Lang, 2007. 
Borrero SILVA, M ${ }^{\text {a }}$ del Valle. «El proceso colonizador en Sonora. La población civil y presidencial en un ambiente de frontera». Orbis incognitvs: avisos y legajos del Nuevo Mundo. Ed. Fernando Navarro Antolín, vol. 2. Huelva: Servicio de Publicaciones de la Universidad de Huelva, 2008. 243-248.

Bullón Fernández, María, ed. England and Iberia in the Middle Ages, 12th-15th Century. Cultural, Literary and Political Exchanges. England: Palgrave MacMillan, 2007.

CALDERÓN CUADRADO, Reyes. «Alianzas comerciales hispano-norteamericanas en la financiación del proceso de independencia de los Estados Unidos de América: la Casa Gardoqui e hijos». Norteamérica a finales del siglo XVIII: España y los Estados Unidos. Ed. Emma Sánchez Montañés, Sylvia L. Hilton, Almudena Hernández Ruigómez e Isabel García-Montón. Coord. Eduardo Garrigues López-Chicheri. Madrid: Fundación Consejo España-Estados Unidos y Marcial Pons, 2008. 197-218.

Carrera, María José, Carrera, Anunciación, Cámara, Enrique y Dapia, Celsa, eds. The Irish Knot: Essays on Imaginary/Real Ireland. Valladolid: Secretariado de Publicaciones e Intercambio Editorial, 2008.

DIN, Gilbert D. «El Alto Mississippi en el último tercio del siglo XVIII: encrucijada de culturas y núcleo estratégico en la rivalidad colonial». Norteamérica a finales del siglo XVIII: España y los Estados Unidos. Ed. Emma Sánchez Montañés, Sylvia L. Hilton, Almudena Hernández Ruigómez e Isabel García-Montón. Coord. Eduardo Garrigues López-Chicheri. Madrid: Fundación Consejo España-Estados Unidos y Marcial Pons, 2008. 83-98.

DownING, Angela. «From Transparency to Opacity: Surely, Intersubjetivity and English Cultural Norms». Proceedings 31st AEDEAN Conference. Ed. María Jesús Lorenzo Modia. A Coruña: Universidade da Coruña, Servizo de Publicacións, 2008. CD-ROM.

FERNÁNDEZ AMAYA, Lucía. «Teaching culture: is it possible to avoid pragmatic failure?». Revista Alicantina de Estudios Ingleses 21 (Nov. 2008): 11-24.

FERNÁNDEZ CANEDO, Virginia. «From the Periphery into the Centre, or From Contemptible Rebel to Epical Figure: Roger Williams and the New England Way». Periphery and Centre III. Eds. Rubén Jarazo Álvarez y Lidia María Montero Ameneiro. A Coruña: Nino, 2008. 29-36.

FERrús ANTÓN, Beatriz. Sor María de Ágreda: historia y leyenda de la dama azul en Norteamérica. Valencia: Publicacions de la Universitat de Valencia, Biblioteca Javier Coy d'estudis Nord-americans, 2008.

García-Montón García-BAQuero, $\mathrm{M}^{\mathrm{a}}$ Isabel. «Aportaciones hispánicas al conocimiento de Estados Unidos». De ida y vuelta América y España: los caminos de la cultura. Eds. Pilar Cagiao Vila y Eduardo Rey Tristán. Santiago de Compostela: Edicion Servizo Publicacións e Intercambio Científico, Universidad Santiago de Compostela, 2007. 293-308.

GodED RAMBAUD, Margarita. «La percepción sensorial y el léxico de la comida y la bebida en inglés y español». Estudios de Filología Inglesa. Homenaje a la Dra. Asunción Alba Pelayo. Ed. Teresa Gibert Maceda y Laura Alba Juez. Madrid: UNED, 2008. 163-185.

Gómez GonZÁlez, Ma de los Ángeles, Mackenzie, J. Lachlan y GonZÁlez Álvarez, Elsa Maa eds. Languages and Cultures in Contrast and Comparison. Amsterdam: John Benjamins Publishing Co., 2008.

Gómez Souto, Luis. «Algunhas crenzas sobre cabalos demoníacos en Galicia, Bretaña e Irlanda». Periphery and Centre III. Eds. Rubén Jarazo Álvarez y Lidia María Montero Ameneiro. A Coruña: Nino, 2008. 11-18.

GonZÁlez CruZ, María Isabel, coord. Lengua, sociedad y cultura: estudios interdisciplinares. Las Palmas de Gran Canaria: Servicio de Publicaciones de la ULPGC/ La Caja de Canarias, 2006.

GONZÁLEZ CRUZ, David. «Represión y trato a los indígenas enemigos en la América hispana durante los enfrentamientos armados del siglo XVIII». Orbis incognitvs: avisos y legajos del Nuevo 
Mundo. Ed. Fernando Navarro Antolín, vol. 2. Huelva: Servicio de Publicaciones de la Universidad de Huelva, 2008. 235-242.

GonZÁLEZ Morales, Belén. «El archipiélago en las periferias: entre Carrasco de Figueroa y Walcott». Periphery and Centre III. Eds. Rubén Jarazo Álvarez y Lidia María Montero Ameneiro. A Coruña: Nino, 2008. 133-141.

González RodríGUez, Ma Paz y Borrero LóPez, Raquel. « El americanismo en la Universidad de Extremadura». Orbis incognitvs: avisos y legajos del Nuevo Mundo. Ed. Fernando Navarro Antolín, vol. 2. Huelva: Servicio de Publicaciones de la Universidad de Huelva, 2008. $943-$ 952.

GUERra Gómez, Amparo. La nueva imagen democrata con Jimmy Carter. Liderazgo presidencial y estrategias electorales para el siglo XXI. Málaga: Sepha Editorial, 2008.

Guardia Herrero, Carmen de la. «La Corte de Madrid y la virtud republicana: los escritos de Sarah Livingston Jay (1779-1784). Norteamérica a finales del siglo XVIII: España y los Estados Unidos. Ed. Emma Sánchez Montañés, Sylvia L. Hilton, Almudena Hernández Ruigómez e Isabel García-Montón. Coord. Eduardo Garrigues López-Chicheri. Madrid: Fundación Consejo España-Estados Unidos y Marcial Pons, 2008. 259-280.

Hilton, Sylvia L. «'Un pueblo que aumentará a pérdida de vista': tempranas impresiones españolas sobre los Estados Unidos». Norteamérica a finales del siglo XVIII: España y los Estados Unidos. Ed. Emma Sánchez Montañés, Sylvia L. Hilton, Almudena Hernández Ruigómez e Isabel García-Montón. Coord. Eduardo Garrigues López-Chicheri. Madrid: Fundación Consejo España-Estados Unidos y Marcial Pons, 2008. 237-257.

Hobson, Fred. A Southern Enigma: Essays on the U.S. South. Valencia: Publicacions de la Universitat de València, Biblioteca Javier Coy d'estudis Nord-Americans, 2008.

JAIME DE PABLos, María Elena. Análisis de género en los estudios irlandeses. Almería: Servicio de Publicaciones de la Universidad de Almería, 2007.

Jarazo Álvarez, Rubén y Montero Ameneiro, Lidia María, eds. Periphery and Centre III. A Coruña: Nino, 2008.

LAMIKIZ, Xavier. «El impacto del 'libre comercio' con América: una revisión desde la microhistoria (1778-1796)». Orbis incognitvs: avisos y legajos del Nuevo Mundo. Ed. Fernando Navarro Antolín, vol. 2. Huelva: Servicio de Publicaciones de la Universidad de Huelva, 2008. 189-197.

Lojo SANDino, Paula. «Language Contact on the Atlantic Periphery: Ireland and Galicia». Periphery and Centre III. Eds. Rubén Jarazo Álvarez y Lidia María Montero Ameneiro. A Coruña: Nino, 2008. 19-27.

LóPEz CHÁvez, Celia. «Benjamín Franklin y la realeza española: una conexión musical». Norteamérica a finales del siglo XVIII: España y los Estados Unidos. Ed. Emma Sánchez Montañés, Sylvia L. Hilton, Almudena Hernández Ruigómez e Isabel García-Montón. Coord. Eduardo Garrigues López-Chicheri. Madrid: Fundación Consejo España-Estados Unidos y Marcial Pons, 2008. 127-141.

LóPEZ ZAPICO, Misael Arturo. Las relaciones entre Estados Unidos y España durante la guerra civil y el primer franquismo (1936-1945). Gijón: Ediciones TREA, S.L., 2008.

Lorenzo Modía, Ma Jesús y Boland, Roy C. Australia and Galicia. Defeating the Tyranny of Distance. Sydney: Antípodas Monographs, Xunta de Galicia, 2008.

LUCENA GIRALDO, Manuel. «La expansión urbana hispánica, de Florida a California». Norteamérica a finales del siglo XVIII: España y los Estados Unidos. Ed. Emma Sánchez Montañés, Sylvia L. Hilton, Almudena Hernández Ruigómez e Isabel García-Montón. Coord. Eduardo Garrigues López-Chicheri. Madrid: Fundación Consejo España-Estados Unidos y Marcial Pons, 2008. 29-43.

Martín Alegre, Sara, ed. Recycling Culture(s). New Castle: Newcastle Scholars Publishing, 2008. 
MONRÓS GASPAR, Laura. «Mythic Women in Victorian England. Cassandra and Florence Nightingale». Proceedings 31st AEDEAN Conference. Ed. María Jesús Lorenzo Modia. A Coruña: Universidade da Coruña, Servizo de Publicacións, 2008. 169-180. CD-ROM.

MonTES, Catalina. «La España decimonónica vista por un inglés». Estudios de Filología Inglesa. Homenaje a la Dra. Asunción Alba Pelayo. Ed. Teresa Gibert Maceda y Laura Alba Juez. Madrid: UNED, 2008. 471-489.

Mora, María José, Gómez-Lara, Manuel, Portillo, Rafael y Prieto-Pablos, Juan A. eds. The woman turned bully. Barcelona: Publicacións i Edicións de la Universitat de Barcelona, 2007.

Moreno Alonso, Manuel. Las cosas de España en Inglaterra. Un país ante la mirada del otro. Sevilla: Ediciones Alfar, 2007.

Muñoz Calvo, Micaela, Buesa Gómez, Carmen y Ruiz Moneva, Ma Ángeles, eds. New Trends in Translation and Cultural identity. New Castle. Cambridge Scholars Publishing, 2008.

NIÑo, Antonio. «El exilio de 1939 y la movilización estadounidense en favor de los académicos españoles». Exils, passages et transitions. Chemins d'une recherche sur les marges. Clermont-Ferrand : Presses Universitaires Blaise Pascal, 2008. 73-83.

-. «El exilio intelectual republicano en los Estados Unidos». Cuadernos de Historia Contemporánea, número extraordinario (2007): 229-244.

OlIETE, Elena. «Centre and Periphery in British Cultural Identity: Representations of Intercultural Relationships in David Lean's A Passage to India». Periphery and Centre III. Eds. Rubén Jarabo Álvarez y Lidia María Montero Ameneiro. A Coruña: Nino, 2008. 47-55.

Pascual, Nieves, Alonso-Gallo, Laura y Collado-Rodríguez, Francisco, eds. Masculinities, Feminities and the Power of the Hybrid in U.S. Narratives: Essays on Gender Borders. Heidelberg: Universitätsverlag, 2007.

PAZos VÁzQUEZ, Paula. «Feminists of Colour Redefine Difference: Trinh T. Minh-ha and Gloria Anzaldúa». Periphery and Centre III. Eds. Rubén Jarazo Álvarez y Lidia María Montero Ameneiro. A Coruña: Nino, 2008. 113-131.

Pérez Murillo, Ma Dolores. «Americanismo y oralidad en la Universidad de Cádiz desde 1992 a 2007». Orbis incognitvs: avisos y legajos del Nuevo Mundo. Ed. Fernando Navarro Antolín, vol. 2, Huelva: Servicio de Publicaciones de la Universidad de Huelva, 2008. 935942.

PÉREZ SAMPER, Ma Ángeles. «Franklin y Aranda: dos embajadores en París». Norteamérica a finales del siglo XVIII: España y los Estados Unidos. Ed. Emma Sánchez Montañés, Sylvia L. Hilton, Almudena Hernández Ruigómez e Isabel García-Montón. Coord. Eduardo Garrigues López-Chicheri. Madrid: Fundación Consejo España-Estados Unidos y Marcial Pons, 2008. 101-125.

PIQUERAS FraILE, $M^{\text {a }}$ del Rosario. «La esclava negra americana del siglo XIX, la gran marginada». De lo sagrado y lo profano. Mujeres tras/entre/sin fronteras. Ed. Mercedes Arriaga Flórez et al. Sevilla: Arcibel Editores, 2008. 313-325.

RÁBADE LÓPEZ, Paula. «Scotland, Ireland and England. A Troubled Web». Periphery and Centre III. Eds. Rubén Jarazo Álvarez y Lidia María Montero Ameneiro. A Coruña: Nino, 2008. 67-89.

RODRÍGUEZ GoNZÁLEZ, Carla. «Rasgos autobiográficos en la poesía de la diáspora escocesa: la obra de Gerrie Fellows». Feminismos e Interculturalidad. Ed. Mercedes Arriaga Flórez et al. Sevi1la: Arcibel Editores, 2008. 367-379.

SAGARRA GAMAZO, Luis. «Una imagen para una utopía: los Estados Unidos en el semanario chaqueño El Colono (1906-1922». Orbis incognitvs: avisos y legajos del Nuevo Mundo. Ed. Fernando Navarro Antolín, vol. 2. Huelva: Servicio de Publicaciones de la Universidad de Huelva, 2008. 633-640.

SAGREDo SANTOS, Antonia. «Imágenes de la revolución americana y de la época formativa de los Estados Unidos en la historiografía española». Orbis incognitvs: avisos y legajos del Nuevo 
Mundo. Ed. Fernando Navarro Antolín, vol. 2. Huelva: Servicio de Publicaciones de la Universidad de Huelva, 2008. 301-308.

- . «An Excepcional First Lady and her role in Politics in the 20th century. Eleanor Roosevelt». Estudios de Filología Inglesa. Homenaje a la Dra. Asunción Alba Pelayo. Ed. Teresa Gibert Maceda y Laura Alba Juez. Madrid: UNED, 2008. 593-602.

-. «La ley de Reforma Agraria de 1932 y la Agricultural Adjustment Act de 1933: Paralelismos y divergencias de dos leyes agrarias liberales». Movimientos Sociales de la España Contemporánea. Ed. Antonio Rivera, José $\mathrm{M}^{\mathrm{a}}$ Ortiz de Ortuño y Javier Ugarte. Madrid. Abada Editores, 2008. 543-568.

- . Mujeres marginadas y perseguidas por sus creencias religiosas en el periodo colonial norteamericano». De lo sagrado y lo profano. Mujeres tras/entre/sin fronteras. Ed. Mercedes Arriaga Flórez et al. Sevilla: Arcibel Editores, 2008. 421-432.

- . «Mujeres que protagonizaron el periodo revolucionario estadounidense». Feminismos e Interculturalidad. Ed. Mercedes Arriaga Flórez et al. Sevilla: Arcibel Editores, 2008. 401-413.

- . Piqueras Fraile, $M^{a}$ del Rosario y Arroyo Vázquez, $M^{a}$ Luz. «Recordando a mujeres relevantes de la historia y cultura escocesa». Proceedings 31st AEDEAN Conference. Ed. María Jesús Lorenzo Modia. A Coruña: Universidade da Coruña, Servizo de Publicacións, 2008. CDROM.

SÁNCHEZ MonTAÑÉs, Emma «Fuentes españolas y etnografía. La costa pacífica estadounidense a finales del siglo XVIII». Norteamérica a finales del siglo XVIII: España y los Estados Unidos. Ed. Emma Sánchez Montañés, Sylvia L. Hilton, Almudena Hernández Ruigómez e Isabel García-Montón. Coord. Eduardo Garrigues López-Chicheri. Madrid: Fundación Consejo EspañaEstados Unidos y Marcial Pons, 2008. 45- 68.

- , Hilton, Sylvia L., Hernández Ruigómez, Almudena y García-Montón, Isabel, eds. Norteamérica a finales del siglo XVIII: España y los Estados Unidos. Coordinador: Eduardo Garrigues López-Chicheri. Madrid: Fundación Consejo España-Estados Unidos y Marcial Pons, 2008.

SÁNCHEZ SuÁREZ, M ${ }^{a}$ Eugenia. «La infancia de Maya Angelou en el sur de Estados Unidos y su exilio voluntario». De lo sagrado y lo profano. Mujeres tras/entre/sin fronteras. Eds. Mercedes Arriaga Flórez et al. Sevilla: Arcibel Editores, 2008. 461-470.

SANTIAGo CAZULL, Héctor. «Conflicto, alianza y disociación en el Puerto Rico del siglo XVII: las redes sociales del capitán general Sancho Ochoa de Castro y el sargento mayor Jerónimo de Mieses (1602-1608)». Revista Complutense de Historia de América 34 (2008): 43-62.

Thomas, Joan María. Roosevelt y Franco. Barcelona: Edhasa, 2007.

UngERER, Gustav. The Mediterranean Apprenticeship of British Slavery. Madrid: Verbum, 2008.

VALdÉs, Miyares, Rubén y RodríguEz GonZÁLEZ, Carla, eds. Cultura and Power: The Plots of History in Performance. Newcastle: Cambridge Scholars Publishing, 2008.

VAlera-ZAPATA, Jesús. «Minority Diasporas and Migration: Fray Rosendo Salvado as a Pioneer of Spanish and Galician Settlement in Australia». Proceedings 31st AEDEAN Conference. Ed. María Jesús Lorenzo Modia. A Coruña: Universidade da Coruña, Servizo de Publicacións, 2008. CD-ROM.

VAlero Garcés, Carmen y MARTín, Anne, eds. Crossing Borders in Community Interpreting: Definitions and Dilemmas. Amsterdam \& Philadelphia: John Benjamins Library, 2008.

WoOdWARd SMITH, Elizabeth, ed. Approaching Cultures through English. A Coruña: Servicio de Publicacións da Universidade de A Coruña, 2007. 


\section{CINE}

Alberola, Nieves y Shafer, Yvonne, eds. ¿Nimiedades para la eternidad?. Pioneras en la escena estadounidense. Castellón: Eliago Ediciones, 2006.

Alonso, Juan J. «La Edad Media según Hollywood: de Robin Hood a las cruzadas». Clío: Revista de historia 69 (2007): 36-43.

ARIAS, Alfredo. «Disney, tenemos un problema». Revista Latente: revista de historia y estética del audiovisual 5 (2007): 205-216.

BernaL, María. «Verano al abordaje: hablamos con Johnny Depp, Keira Knightley, Orlando Bloom y el director Gore Verbinski». Fotogramas \& DVD: La primera revista de cine 1954 (2006): 64-71.

BLÁNQUEZ, Javier. «Lo último del cine fantástico». Fotogramas \& DVD: La primera revista de cine 1962 (2007): 104-107.

Bou, Núria. Diosas y tumbas: mitos femeninos en el cine de Hollywood. Barcelona : Icaria, 2006.

CAPARRós Lera, J. M. Woody Allen, Barcelonés accidental sólo detrás de la cámara. Madrid: Encuentro, 2008.

CARMONA, Luis Miguel. 200 diosas menores del cine a recordar. Madrid: Cacitel, S. L., 2008.

CARRERA SUÁREZ, Isabel. «El rostro femenino de Europa: Casablanca, Ingrid Bergman y el orientalismo del cine clásico de Hollywood». Diosas del celuloide: arquetipos de género en el cine clásico. Coord. Carmen Rodríguez Fernández. Ediciones Jaguar, 2007. 45-66.

Casas Guijarro, Manuel. «The (In)sides Beyond Male Friendship-Desire: Alterity and Minortities in Michael Radford's Merchant of Venice (2004)». Proceedings 31st AEDEAN Conference. Ed. María Jesús Lorenzo Modia. A Coruña: Universidade da Coruña, Servizo de Publicacións, 2008. CD-ROM.

CASTRO, Genoveva. «Bollywood: un Hollywood con ocho brazos». Letras libres 106 (2007): 95-96.

CAscajosa Virino, Concepción. «De la TV a Hollywood: un repaso a las películas basadas en series». Madrid : Arkadin, 2006.

- . El espejo deformado: versiones, secuelas y adaptaciones en Hollywood. Sevilla: Secretariado de Publicaciones, Universidad de Sevilla, 2006.

ChAKRABARTI, Raita Merivirta. «Between Irish National Cinema and Hollywood: Neil Jordan's Michael Collins». Estudios irlandeses. Journal of Irish Studies 2 (2007): 121-127.

CoHEN, Clélia. El western: el cine americano por excelencia, la conquista del Oeste, el nacimiento de un género, artistas y modelos: Ford, Wayne, Mann, Stewart. Barcelona: Paidós Ibérica, 2006.

ComA, Javier. «Circo para el púlpito: Elmer Gantry / El fuego y la palabra». Revista de cine 382 (2008): 70-77.

—. «Canciones en la obra de Ford: un código íntimo de signos». Revista de cine 380 (2008): 58-62.

—. «Caza de brujas: films antisoviéticos al comenzar la guerra fría». Revista de cine 370 (2007): 58-65.

-. «La venganza es la ruleta: los 'westerns' de un crupier de la fatalidad». Revista de cine 367 (2007): 84-87.

-. «Piratas del mar Caribe: Cecil B. De Mille, 1942». Revista de cine 356 (2006). 82-83.

Cominges, Jorge de. «Navidades blancas». Fotogramas \& DVD: La primera revista de cine 1958 (2006): 182-186.

Cruzado Rodríguez, Ángeles. «Grietas en el techo del celuloide. Directoras del siglo XXI». Mujeres, espacio y Poder. Ed. Mercedes Arriaga Flórez, et al. Sevilla: Arcibel Editores, 2006. 153-173.

-. «Ellos las prefieren sumisas. La feminidad ideal vista por el cine». De lo sagrado y lo profano. Mujeres tras/entre/sin fronteras. Ed. Mercedes Arriaga Flórez et al. Sevilla: Arcibel Editores, 2008. 95-104. 
DIEZ, Emeterio. «Censura franquista en Hollywood. Por quién doblan las campanas». Historia 16, 386 (Junio 2008): 84-105.

ERwIN, Ellen y Diamond, Jessica Z. Los tesoros de Audrey Hepburn. Barcelona: Editorial Cúpula, 2008.

FERNÁNDEZ, Fausto. «Philip Seymour Hoffman: del estrellato Indie al glamour de Hollywood». Fotogramas \& DVD: La primera revista de cine 1951 (2006): 102-104.

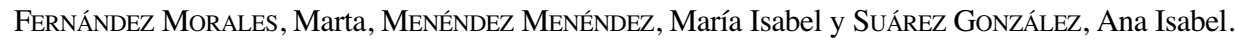
Seré breve... Guía didáctica para el análisis y explotación de cortometrajes desde la perspectiva de género. Oviedo: Milenta, 2008.

Fernandez VALENTI, Tomás. Martin Scorsese: un infiltrado en Hollywood. Barcelona. Ediciones Carena, 2008.

FERro, Marc. El cine, una visión de la historia. Madrid: Akal, 2008.

FEZ, Desirée de. «Hollywood made in Spain: cine de género 100 por cien exportable». Fotogramas \& DVD: La primera revista de cine 1970 (2007): 112-113.

FINLER, Joel W. Historia de Hollywood. Teià , Barcelona: Ma Non Troppo, 2006.

FloRes Juberías, Carlos, ed. Todos los filmes del presidente. (La presidencia de los Estados Unidos vista a través del cine). Valencia: Museo Valencià de la Il-lustració i de la Modernitat. Area de Cultura de la Diputació de Valencia, 2008.

FonT, Carmen. «Born in the Streets: Violence, Gansterism and Power in Gangs of New York». Proceedings 31st AEDEAN Conference. Ed. María Jesús Lorenzo Modia. A Coruña: Universidade da Coruña, Servizo de Publicacións, 2008. CD-ROM.

FONT, Oscar. That old feeling: la historia de la Woody Allen's New Orleans funeral and ragtime orchestra (1972-1996). Barcelona: Autor-Editor, 2007.

Frías CASTILLEJo, Carolina. «Espartaco y la lucha de clases en el Hollywood de McCarthy». Quaderns de cine 1 (2007): 61-68.

GómeZ TARín, Francisco Javier. Wong Kar-Wai. Madrid: Akal, 2008.

GonZALEZ, Palmira. El cine de animación norteamericano / El cine mudo. Barcelona: Editorial UOC. 2008.

González Requena, Jesús. Clásico, manierista, postclásico : los modos del relato en el cine de Hollywood. Valladolid : Castilla, 2006.

GorostiZA, Jorge. La profundidad de la pantalla «arquitectura+cine». Madrid: Ocho y Medio, 2007.

JAIME, Graham. «Paul Thomas Anderson». Cinemanía 149 (2008): 68-72.

GUARDIA CALVO, Isadora. «La clase obrera: objeto representado, sujeto negado en el texto fílmico». Movimientos Sociales de la España Contemporánea. Ed. Antonio Rivera, José $\mathrm{M}^{\mathrm{a}} \mathrm{Ortiz}$ de Ortuño y Javier Ugarte. Madrid. Abada Editores, 2008. 323-333.

GuILlÉn, Sergio. 80 peliculas de los 80. Madrid: T\&B Editores, 2008.

HUERTA Floriano, Miguel Ángel. «Cine y política de oposición en la producción estadounidense tras el 11-S». Comunicación y sociedad: Revista de la Facultad de Comunicación (Universidad de Navarra) 21.1 (2008): 81-102.

IgLesias, Eulalia. «Forest Whitaker: un talento enorme». Fotogramas \& DVD: La primera revista de cine 1961 (2007): 100-101.

LAmarCa, Manuel. Cómo crear una película. Madrid: T\&B Editores, 2008.

LATORRE, Jorge. «Blade Runner forever». Nuestro tiempo 646 (2008): 103-110.

Louvish, Simon. Money business, vida y leyenda de los hermanos Marx. Madrid: T\&B Editores, 2008.

MARAÑón, Carlos. «Dalí goes to Hollywood». Cinemanía 164 (2009): 36-37.

MÉNDEZ Domínguez, Mario. «Hollywood les presenta: Andalucía». Frame 1 (2007): 219-225.

MIRÓN, Cándido. «Y Hollywood enseñó las piernas». Fotogramas \& DVD: La primera revista de cine 1978 (2008): 100-104. 
MARTín Ayuso, Ma Isabel. «'If You Can’t Have a Friend, Make One'. Lucky McKee's May as a revision of Mary Shelley's Frankenstein». Proceedings 31st AEDEAN Conference. Ed. María Jesús Lorenzo Modia. A Coruña: Universidade da Coruña, Servizo de Publicacións, 2008. CDROM.

MARTín GutiérRez, Gregorio, coord. Cineastas frente al espejo. Madrid: T\&B Editores, 2008.

Martín Renes, Cornelis. «Tempted by the Tempest: Dereck Jarman's Gay Play with Shakespearean Romance». Proceedings 31st AEDEAN Conference. Ed. María Jesús Lorenzo Modia. A Coruña: Universidade da Coruña, Servizo de Publicacións, 2008. CD-ROM.

MarTínez-CABeZa Lombardo, Miguel Angel . «'Based on a true story»: Spanish cinema looks at counter-terrorism». Studies in Honour of Neil Mclaren: A Man for all Seasons. Coord. Angeles Linde López, Juan Santana Lario y Celia Margaret Wallhead Salway. Granada: Editorial Universidad de Granada, 2008. 297-306.

Memba, Javier. Historia del cine universal. Madrid: T\&B Editores, 2008.

Mena, José Luis. Los Óscars de 2007. Madrid: Cacitel, S. L., 2008.

MolDES, Diego. El cine europeo: las grandes películas. Madrid: Ediciones JC Clementine, 2008.

MonTOYA, Alex. «El cine y la vida según Howard Hawks». Fotogramas \& DVD: La primera revista de cine 197 (2007): 188-193.

- . «Tocado y hundido». Fotogramas \& DVD: La primera revista de cine 1954 (2006): 78-81.

Natham Ditum, Yago García. Hollywood 2.0. Cinemanía 146 (2007): 60-69.

NAVARro, Antonio José. «Daños colaterales: la 'generación de la violencia' y el cine de Hollywood». Nosferatu: Revista de cine 53-54 (2006) (Ejemplar dedicado a la 'generación de la violencia' del cine norteamericano) Coord. José María Latorre): 82-92.

NoAín, Idoya. «Syriana: Clooney manchado por el oro negro». Fotogramas \& DVD: La primera revista de cine 1949 (2006): 110-113.

OCAÑA, Javier. «El diablo sabe que está vivo». Cinemanía 148 (2008): 33.

Ortega, M ${ }^{\mathrm{a}}$ Luisa y Noemí García. Cine Directo: reflexiones en torno a un concepto. Madrid: T\&B Editores, 2008.

OTERo, Mercé. «Luk Sky Biskind Vs. Darth Harvey». Cinemanía 138 (2007): 132.

Palacios GonZÁlez, Manuela y GonZÄlez FernándeZ, Helena, eds. Palabras extremas: escritoras gallegas e irlandesas de hoy. A Coruña: Netbiblo (Irish Studies Series), 2008.

PALENCIA, Leandro. Hollywood Queer. Madrid: T\&B Editores, 2008.

PAvÉs, Gonzalo M. «Bailando nace el Amor: el cortejo amoroso en el cine musical de Hollywood». Boletín de arte 29 (2008): 455-480.

Pelaz LóPEz, José Vidal. «Cae el telón. El cine norteamericano en los inicios de la Guerra Fría (1945-1954)». Historia Actual Online 15, (2008): 125-136.

Perales BAZo, Francisco. «La geografía andaluza como plató hollywoodiense». Frame 1 (2007): 226-235.

PÉREZ BowIE, José Antonio. Leer el cine. La teoría literaria en la teoría cinematográfica. Salamanca: Universidad de Salamanca, 2008.

PéreZ Niño, Tomás. Diosas del cine para adultos. Madrid: Capitel D. L., 2008.

PhILIPS, Dave. «La magia del cine: cita con el reproductor de medios Tótem». Linux magazine 44 (2008) : 70-72.

Ponga, Paula. «S.O.S. Indies en peligro de muerte». Fotogramas \& DVD: La primera revista de cine 1982 (2008): 154-160.

Puente Gómez, Andrés y Guillén BarRantes, Sergio. 80 películas de los 80: una lectura ácida. Madrid: T\&B Editores, 2008.

RAmos Rodríguez, María José. «Cine y moda en Hollywood: décadas de los cuarenta y cincuenta». Revista Latente: revista de historia y estética del audiovisual 5 (2007): 183-204.

RENTERo, Juan Carlos. Todos los estrenos de 2008. Madrid: Ediciones JC Clementine, 2008. 
ReQuena, Jesús González. Clásico, manierista, postclásico : los modos del relato en el cine de Hollywood. Valladolid : Castilla, 2006.

RodRíGUEZ MARTíN, María Elena y RodRíGuEz SALAS, Gerardo. «Introducing temporal relations through novel and film: Michael Cunningham's The Hours and its film adaptation». Studies in Honour of Neil Mclaren: A Man for all Seasons. Coord. Angeles Linde López, Juan Santana Lario y Celia Margaret Wallhead Salway. Granada: Editorial Universidad de Granada, 2008. $339-50$.

ROMERo, Rubén. «Los 7 mandamientos de David Fincher». Cinemanía 140 (2007): 76-80.

SALVAns, Roger. «60 años de stars». Fotogramas \& DVD: La primera revista de cine 1964 (2007): 154-157.

SÁNCHEZ, Sergi. «Hollywood la meca de los horrores». Fotogramas \& DVD: La primera revista de cine 1967 (2007): 92-97.

-. «Festival de Sundance: ¿independientes de qué?». Fotogramas \& DVD: La primera revista de cine 1961 (2007): 110-114.

—. «A esos grandes desconocidos». Fotogramas \& DVD: La primera revista de cine 1962 (2007): 10.

SCHULBERG, Budd. De cine: memorias de un príncipe de Hollywood. Barcelona : Acantilado, 2006.

SiKov, Ed. Betty Davis. Amarga victoria. Madrid. T \& B Editores, 2008.

Simsolo, Noel y Martorell Linares, Alicia, trad. El cine negro: Pesadillas verdaderas y falsas. Madrid: Alianza Editorial, 2007.

TEJERO, Juan. John Wayne, el vaquero que conquisto Hollywood. Madrid: T \& B Editores, 2008.

TORRES ZÚÑIGA, Laura. «Feminist Commentary in Film Adaptation: an Analysis of Shelmerdine in Rally Potter's Orlando». Periphery and Centre III. Eds. Rubén Jarabe Álvarez y Lidia María Montero Ameneiro. A Coruña: Nino, 2008. 37-46.

Valantin, Jean Michel. Hollywood, el Pentágono y Washington: los tres actores de una estrategia global. Barcelona: Alertes, 2008.

VV.AA. Orígenes del cine (2 vols.). Madrid: Divisa Red, 2008.

VILLAR ARGÁIz, Pilar. «Latter-day Mother Irelands: The Role of Women in Michael Collins and The Wind that Shakes the Barley». Estudios irlandeses. Journal of Irish Studies 2 (2007): 183-204.

VILLAR Flor, Carlos. «The Symbols of Music in John Huston's The Dead». The Irish Knot: Essays on Imaginary / Real Ireland. Ed. María José Carrera, Anunciación Carrera, Enrique Cámara, Celsa Dapía. Valladolid: Universidad de Valladolid, 2008. 127- 137.

VIÑUELA SUÁREZ, Eduardo. «El estudio del videoclip desde la perspectiva de género». Feminismos e Interculturalidad. Ed. Mercedes Arriaga Flórez et al. Sevilla: Arcibel Editores, 2008. 483494.

WILlis, Jaime. Warner Bros. Madrid: T\&B Editores, 2008.

ZuMALDE ARREGUI, Imanol. «Estamp(it)as multiculturales. Las tribulaciones del texto fílmico contemporáneo ante las veleidades de la crítica posmoderna». Zer: Revista de estudios de comunicaci / Komunikazio ikasketen aldizkaria 24 (2008): 223-236. 